\title{
Secure Degrees of Freedom of MIMO Rayleigh Block Fading Wiretap Channels With No CSI Anywhere
}

\author{
Ta-Yuan Liu, Student Member, IEEE, Pritam Mukherjee, Student Member, IEEE, Sennur Ulukus, Member, IEEE, \\ Shih-Chun Lin, Member, IEEE, and Y.-W. Peter Hong, Senior Member, IEEE
}

\begin{abstract}
We consider the block Rayleigh fading multipleinput multiple-output (MIMO) wiretap channel with no prior channel state information (CSI) available at any of the terminals. The channel gains remain constant within a coherence interval of $T$ symbols, and then change to another independent realization in the next coherence interval. The transmitter, the legitimate receiver, and the eavesdropper have $n_{t}, n_{r}$, and $n_{e}$ antennas, respectively. We determine the exact secure degrees of freedom (s.d.o.f.) of this system when $T \geq 2 \min \left(n_{t}, n_{r}\right)$. We show that, in this case, the s.d.o.f. is exactly equal to $\left(\min \left(n_{t}, n_{r}\right)-n_{e}\right)^{+}\left(T-\min \left(n_{t}, n_{r}\right)\right) / T$. The first term in this expression can be interpreted as the eavesdropper with $n_{e}$ antennas taking away $n_{e}$ antennas from both the transmitter and the legitimate receiver. The second term can be interpreted as a fraction of the s.d.o.f. being lost due to the lack of CSI at the legitimate receiver. In particular, the fraction loss, $\min \left(n_{t}, n_{r}\right) / T$, can be interpreted as the fraction of channel uses dedicated to training the legitimate receiver for it to learn its own CSI. We prove that this s.d.o.f. can be achieved by employing a constant norm channel input, which can be viewed as a generalization of discrete signalling to multiple dimensions.
\end{abstract}

Index Terms-Physical layer secrecy, wiretap channel, secure degrees of freedom, multiple-input multiple-output (MIMO), Rayleigh block fading, non-coherent communications.

\section{INTRODUCTION}

W E consider the wiretap channel where a legitimate transmitter wishes to have information-theoretically secure communication with a legitimate receiver in the presence of an eavesdropper. The wiretap channel was introduced by Shannon [1] for the case of noiseless channels, where it was shown that secure keys and one-time-pad encryption were

Manuscript received May 29, 2014; revised October 21, 2014; accepted January 3, 2015. Date of publication January 9, 2015; date of current version May 7, 2015. This work was supported in part by the Ministry of Science and Technology, Taiwan, under grants 100-2628-E-007-025-MY3 and 101-2221E-011-170-MY3, the Industrial Technology Research Institute, Taiwan, under grant E301AR2900, and US National Science Foundation Grants CNS 0964632, CCF 09-64645, CCF 10-18185, and CNS 11-47811. This work was presented in part at IEEE ICC, Sydney, Australia, June 2014. The associate editor coordinating the review of this paper and approving it for publication was Z. Wang.

T.-Y. Liu and Y.-W. Peter Hong are with the Institute of Communications Engineering, National Tsing Hua University, Hsinchu 30013, Taiwan (e-mail: tyliu@erdos.ee.nthu.edu.tw; ywhong@ee.nthu.edu.tw).

P. Mukherjee and S. Ulukus are with the Department of Electrical and Computer Engineering, University of Maryland, College Park, MD 20742 USA (e-mail: pritamm@umd.edu; ulukus@umd.edu).

S.-C. Lin is with the Department of Electronic and Computer Engineering, National Taiwan University of Science and Technology, Taipei 10607, Taiwan (e-mail: sclin@mail.ntust.edu.tw).

Digital Object Identifier 10.1109/TWC.2015.2390234 necessary for secure communications. The noisy wiretap channel was introduced by Wyner, who determined the capacityequivocation region for the degraded case [2]. Csiszár and Körner generalized his result to arbitrary, not necessarily degraded, wiretap channels [3]. Leung-Yan-Cheong and Hellman determined the capacity-equivocation region of the Gaussian wiretap channel and showed that Gaussian signalling is optimal [4]. The secure degrees of freedom (s.d.o.f.) of the scalar Gaussian wiretap channel is zero.

The multiple-input multiple-output (MIMO) wiretap channel where the legitimate entities and the eavesdropper have multiple antennas was considered for the 2-2-1 case in [5] and the general case in [6]-[8]. These references determined the exact secrecy capacity of the MIMO wiretap channel for the case of full channel state information (CSI) at all terminals, and showed that no channel prefixing is necessary and Gaussian signalling is optimal. It can be deduced from these works that the s.d.o.f. of the MIMO wiretap channel with full CSI is $\min \left(\left(n_{t}-n_{e}\right)^{+}, n_{r}\right)$, where $n_{t}, n_{r}$, and $n_{e}$ are the number of antennas at the transmitter, the legitimate receiver, and the eavesdropper, respectively, and $(x)^{+}=\max (x, 0)$.

The fading wiretap channel with a single antenna at all terminals, where all parties have perfect CSI of all links, was considered in [9]-[12]. Modeling the fading wiretap under full CSI as a bank of independent parallel channels, these references showed that independent Gaussian signalling in all parallel channels, together with water-filling of the total power over these channels, is optimal. Reference [13] considered the single antenna wiretap channel where the transmitter has the legitimate receiver's CSI but no eavesdropper CSI under the assumption of infinite coherence times for channel fading, and showed that Gaussian signalling is optimal in this case. Reference [14] considered the same model under a fast fading condition (single symbol coherence time), and showed that MQAM signalling or Gaussian signalling with added Gaussian artificial noise may outperform plain Gaussian signalling. In the single antenna fading channel, under all CSI conditions, the s.d.o.f. is zero, since it is zero under perfect CSI.

Using multiple antennas at the legitimate users however, nonzero s.d.o.f. may be achieved even under partial CSI conditions. Reference [15] showed that in a MIMO wiretap channel with perfect CSI at the receivers, but only a statistical CSI at the transmitter, under a fast fading Rayleigh channel, the s.d.o.f. of the system is $\left(\min \left(n_{t}, n_{r}\right)-n_{e}\right)^{+}$. Note that this may be 
less than the s.d.o.f. achievable under perfect CSI, which is $\min \left(\left(n_{t}-n_{e}\right)^{+}, n_{r}\right)$. A comparison of these two s.d.o.f. may be interpreted as the eavesdropper taking away $n_{e}$ antennas only from the transmitter in the case of perfect CSI [5]-[8], but $n_{e}$ antennas from both the transmitter and the legitimate receiver in the case of partial CSI [15]. More strongly, reference [16] considered the case of an arbitrarily varying eavesdropper in a MIMO wiretap channel and showed that the same s.d.o.f. of $\left(\min \left(n_{t}, n_{r}\right)-n_{e}\right)^{+}$can be achieved in this case. In [16], the CSI of the legitimate receiver is assumed known at the transmitter, however, nothing is known about the eavesdropper CSI, not even its probability distribution. This is an exceptionally strong modeling of the eavesdropper, where secrecy must be guaranteed for every realization of the eavesdropper channel; in a way, the eavesdropper may be thought to be controlling its channel adversarially.

All of the above work considered that some (either perfect or partial) CSI is available at some of the terminals. In practice, typically, the way CSI becomes available at the terminals is via the receivers measuring it and feeding it back to the transmitters. It is reasonable to assume that no CSI is known at the outset before the start of the communication. One must then take into consideration the cost of acquiring the CSI. In addition, the assumption of perfect CSI is an idealization; in reality, the terminals may only have an estimate of the channel in a delayed manner as discussed in [17]-[19]. Further, in most cases, eavesdropper CSI will not be available at the transmitter, because she will not feed her measurement back, and even if she does, she will not be truthful. Thus, it is more practical to assume that no CSI is available at any terminal a priori. Recently, reference [20] studied the case where no CSI is available at any terminal and the coherence time of the Rayleigh fading channel is one symbol duration. Reference [20] determined the exact secrecy capacity in this case and showed that discrete signalling is optimal. As in all other single antenna cases, the s.d.o.f. in [20] is zero. It can be shown that, even when multiple antennas are added, s.d.o.f. in the case of fast fading in [20] is still zero.

In this paper, we consider the MIMO wiretap channel under block Rayleigh fading, where the channel gains of both the legitimate receiver and the eavesdropper remain fixed for a coherence interval of $T$ symbols, and then change to another independent realization in the next coherence interval. This models a Rayleigh fading wireless communication channel with a coherence time of $T$ symbol durations. We consider the case where neither the transmitter nor the receivers have any CSI. This can be considered as an extension of [20] to the case of multiple antennas and larger (than one) coherence times. A similar channel model without any secrecy constraints was considered in [21], [22], where in [21] the structure of the optimal input distribution was found, and in [22] the degrees of freedom (d.o.f.) was determined to be $m(1-m / T)$ where $m=\min \left(n_{t}, n_{r},\lfloor T / 2\rfloor\right)$. Our work can also be considered as a wiretap version of [21], [22].

We show that when the coherence time $T$ satisfies $T \geq$ $2 \min \left(n_{t}, n_{r}\right)$, the s.d.o.f. of this system is exactly $\left(\min \left(n_{t}, n_{r}\right)-\right.$ $\left.n_{e}\right)^{+}\left(T-\min \left(n_{t}, n_{r}\right)\right) / T$. Compared to the MIMO wiretap channel results in [15], [16], where the legitimate receiver knows its channel gain, the s.d.o.f. in our case is exactly the same as those in [15], [16] except for a factor of $(T-$ $\left.\min \left(n_{t}, n_{r}\right)\right) / T$. Intuitively, at high signal-to-noise ratio (SNR), the legitimate receiver needs $\min \left(n_{t}, n_{r}\right)$ channel uses out of $T$ channel uses to learn its channel. Therefore, the factor $(T-$ $\left.\min \left(n_{t}, n_{r}\right)\right) / T$ intuitively accounts for the number of channel uses lost for estimating the channel at the legitimate receiver. As in the cases of [15], [16], due to no CSI at the transmitter, the eavesdropper takes away $n_{e}$ antennas from both the transmitter and the receiver, i.e., $n_{e}$ is subtracted from $\min \left(n_{t}, n_{r}\right)$, as opposed to being subtracted only from $n_{t}$ as in the case of full CSI at the transmitter [5]-[8]. In comparison to the case without any secrecy constraints in [21], [22], here we have a subtraction of $n_{e}$ from the first term in the d.o.f. due to the presence of the eavesdropper.

Finally, it is interesting to note that one cannot achieve a positive s.d.o.f with either a long coherence time in a single antenna system [13] or with multiple antennas in a very short $(T=1)$ coherence time channel [20]; however, with some moderate coherence $\left(T \geq 2 \min \left(n_{t}, n_{r}\right)\right)$ and use of multiple antennas, it is possible to achieve positive s.d.o.f., as we show in this paper.

\section{System Model And Problem Formulation}

Consider a wiretap channel that consists of a transmitter with $n_{t}$ antennas, a legitimate receiver with $n_{r}$ antennas, and an eavesdropper with $n_{e}$ antennas. The channel between the transmitter and the legitimate receiver is denoted by matrix $\mathbf{H} \in \mathcal{C}^{n_{r} \times n_{t}}$ and the channel between the transmitter and the eavesdropper is denoted by matrix $\mathbf{G} \in \mathrm{e}^{n_{e} \times n_{t}}$. The channels are Rayleigh fading, i.e., entries of the channel matrices are independent and identically distributed (i.i.d.) complex Gaussian random variables with zero-mean and unit-variance denoted by $\mathcal{C N}(0,1)$. The channels are block fading, i.e., the channel coefficients remain constant throughout a coherence interval $T$ and change independently across different intervals according to the same distribution.

Let $\mathbf{X} \in \mathrm{e}^{n_{t} \times T}$ denote the signal transmitted by the transmitter during a coherence interval. The transmitted signal is subject to an average power constraint as,

$$
\frac{1}{T} \mathbb{E}\left[\operatorname{tr}\left(\mathbf{X X}^{\dagger}\right)\right] \leq P
$$

where $\operatorname{tr}(\cdot)$ denotes the trace function. The received signals at the legitimate receiver and the eavesdropper are

$$
\begin{aligned}
& \mathbf{Y}=\mathbf{H X}+\mathbf{N}_{r}, \\
& \mathbf{Z}=\mathbf{G X}+\mathbf{N}_{e},
\end{aligned}
$$

respectively, where $\mathbf{N}_{r} \in \mathcal{C}^{n_{r} \times T}$ and $\mathbf{N}_{e} \in \mathcal{C}^{n_{e} \times T}$ are their respective additive Gaussian noise terms. The entries of $\mathbf{N}_{r}$ and $\mathbf{N}_{e}$ are i.i.d. with distributions $\mathcal{C N}\left(0, \sigma_{r}^{2}\right)$ and $\mathcal{C N}\left(0, \sigma_{e}^{2}\right)$, respectively. The CSI, i.e., the realizations of $\mathbf{H}$ and $\mathbf{G}$, are not known to any of the terminals.

A $\left(2^{n R}, n\right)$ code consists of an encoder $f_{n}$ at the transmitter that maps each secret message, say $W \in \mathcal{W} \triangleq\left\{1, \ldots, 2^{\text {nR }}\right\}$ into 
a length- $n$ codeword and a decoder $g_{n}$ at the legitimate receiver that maps its received signal into a message estimate $\hat{W} \in \mathcal{W}$. Each codeword is transmitted over multiple coherence intervals [21] and $n$ is chosen as a multiple of $T$.

A secrecy rate $R$ is said to be achievable if there exists an encoder $f_{n}$ and a decoder $g_{n}$ such that the probability of error at the legitimate receiver $\mathbb{P}[W \neq \hat{W}]$ goes to zero and the average equivocation at the eavesdropper measured by $\frac{1}{n} H\left(W \mid \mathbf{Z}^{n}\right)$ approaches $\frac{1}{n} H(W)$, as the codeword length $n \rightarrow \infty$, where $\mathbf{Z}^{n}$ denotes the signal received at the eavesdropper over $n$ channel uses. The secrecy capacity $C_{S}$ is the supremum of all such achievable secrecy rates. From [3], the secrecy capacity of the MIMO wiretap channel with no CSI at any terminal is

$$
C_{s}=\frac{1}{T} \max _{V, \mathbf{X}} I(V ; \mathbf{Y})-I(V ; \mathbf{Z}),
$$

where $V$ is an auxiliary random variable that satisfies the Markov chain $V \rightarrow \mathbf{X} \rightarrow \mathbf{Y}, \mathbf{Z}$. Determining the optimal joint distribution of $(V, \mathbf{X})$ and the resulting exact secrecy capacity expression is challenging, instead, in this paper, we focus on determining the s.d.o.f. which is defined as,

$$
D_{s}=\lim _{P \rightarrow \infty} \frac{C_{s}}{\log P} .
$$

The s.d.o.f. characterizes how the secrecy capacity scales with $\log (P)$ for large $P$, i.e., it is the pre-log factor of the secrecy capacity at high SNR.

\section{Summary of the Main Results}

In this section, we first summarize our main results; the proofs will be provided in the following sections. The results are encapsulated in the following lemmas and theorem.

Lemma 1: For the MIMO wiretap channel in (2) and (3), with no CSI at any terminal,

$$
D_{s}=0, \quad \text { if } n_{r} \leq n_{e} .
$$

This implies a negative result that when the eavesdropper has more antennas than the legitimate user, i.e., $n_{r} \leq n_{e}$, the s.d.o.f. $D_{s}$ is always zero. No matter how long the coherence time $T$ is and how many transmitter antennas the system has, the secrecy capacity does not scale with the SNR. However, we show in the following lemmas that a positive s.d.o.f. can be achieved, for $n_{r}>n_{e}$ and $T \geq 2 \min \left(n_{t}, n_{r}\right)$.

Lemma 2: When $n_{r}>n_{e}, n_{r} \leq n_{t}$, and $T \geq 2 n_{r}$, the s.d.o.f. is given by

$$
D_{s}=\left(n_{r}-n_{e}\right)\left(\frac{T-n_{r}}{T}\right) .
$$

Lemma 3: When $n_{r}>n_{e}, n_{r}>n_{t}$, and $T \geq 2 n_{t}$, the s.d.o.f. is given by

$$
D_{s}=\left(n_{t}-n_{e}\right)^{+}\left(\frac{T-n_{t}}{T}\right) .
$$

Lemma 2 considers the case where the transmitter has more antennas than the receiver, whereas Lemma 3 considers the opposite case. Note that, in the latter case, a positivity operator $(\cdot)^{+}$is required since $n_{t}$ may be less than $n_{e}$. We combine the above three lemmas to obtain the following main result of our paper.

Theorem 1: For the MIMO wiretap channel in (2) and (3), with no CSI at any terminal, when $T \geq 2 \min \left(n_{t}, n_{r}\right)$, the s.d.o.f. is given by

$$
D_{s}=\left(\min \left(n_{t}, n_{r}\right)-n_{e}\right)^{+}\left(\frac{T-\min \left(n_{t}, n_{r}\right)}{T}\right) .
$$

Note that when no secrecy constraint is considered, i.e., $n_{e}=0$, the s.d.o.f. in Theorem 1 reduces to the d.o.f. of the noncoherent MIMO Rayleigh block fading channel [22]. Our s.d.o.f. is affected by two factors: the first factor $\left(\min \left(n_{t}, n_{r}\right)-\right.$ $\left.n_{e}\right)^{+}$is the s.d.o.f of the case where perfect CSI is available at the receivers [15] (i.e., where there is no cost due to lack of channel knowledge at the receiver), and the second factor $\left(1-\min \left(n_{t}, n_{r}\right) / T\right)$ reflects the loss in efficiency due to the lack of knowledge of the CSI at the legitimate receiver. One can view the ratio $\min \left(n_{t}, n_{r}\right) / T$ as the cost of channel estimation at the legitimate receiver from the point of view of a training based scheme. Note that, even though Lemmas 2 and 3 (and, thus, Theorem 1) hold only for the case where $T \geq 2 \min \left(n_{t}, n_{r}\right)$, the signalling scheme adopted in their achievability proofs can also be used to derive an achievable s.d.o.f. for the case where $T<2 \min \left(n_{t}, n_{r}\right)$, as given in the following lemma.

Lemma 4: For arbitrary coherence time $T$, the s.d.o.f. satisfies

$$
D_{s} \geq\left(K-n_{e}\right)^{+}\left(\frac{T-K}{T}\right),
$$

where $K=\min \left(n_{t}, n_{r},\left(T+n_{e}\right) / 2\right)$.

Lemma 4 shows that, for given coherence time $T$ and the number of eavesdropper's antennas $n_{e}$, the achievable s.d.o.f. for the case where $T<2 \min \left(n_{t}, n_{r}\right)$ increases with the number of legitimate antennas $n_{t}$ until it reaches $n_{r}$ or $\left(T+n_{e}\right) / 2$. Even though more antennas at the transmitter and the legitimate receiver provides more dimensions for communication, it also implies that more resource is needed to cope with the lack of CSI at the receiver, which is reflected in the term $(T-K) / T$. More details can be found in Section VII.

The above main results can be visualized in Fig. 1. Here, we show the s.d.o.f. of the case with no CSI anywhere (i.e., Theorem 1 and Lemma 4) and compare with that of the case with perfect CSIR but no CSIT (or statistical CSIT) [15]. In Fig. 1(a), the number of receive antennas at the legitimate receiver and the eavesdropper (i.e., $n_{r}$ and $n_{e}$, respectively) and the coherence time $T$ are fixed and are chosen such that $n_{r}>n_{e}$ and $2 n_{r}>T+n_{e}$. By varying the number of transmit antennas $n_{t}$, Theorem 1 shows that the s.d.o.f. with no CSI anywhere is zero when $n_{t} \leq n_{e}$, but increases with the number of transmit antennas when $n_{e}<n_{t} \leq T / 2$. The increase is nonlinear as opposed to the case with perfect CSIR. However, as $n_{t}$ increases beyond $T / 2$, Theorem 1 no longer applies and the achievable s.d.o.f. in Lemma 4 is plotted instead (in dotted line). We can see that the achievable s.d.o.f. continues to increase with $n_{t}$ when $T / 2<n_{t}<\left(T+n_{e}\right) / 2$ and saturates when 


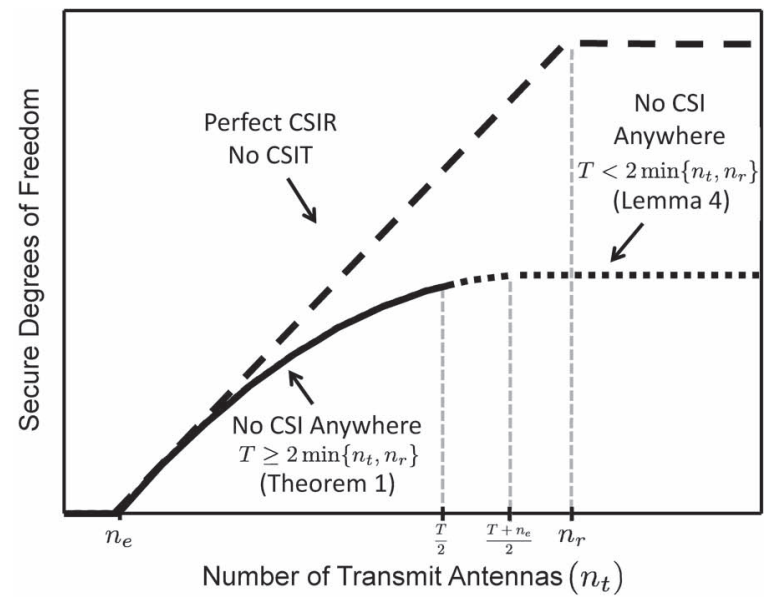

(a)

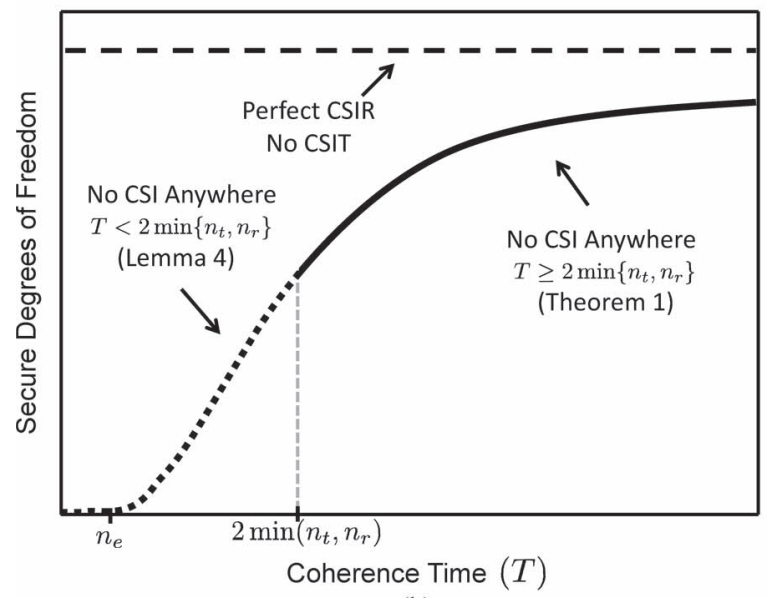

(b)

Fig. 1. Illustrations of the s.d.o.f. derived in Theorem 1 and Lemma 4. (a) The s.d.o.f. versus the number of transmit antennas $n_{t}$ for $n_{r}>n_{e}$ and $2 n_{r}>T+n_{e}$. (b) The s.d.o.f. versus coherence time $T$ for $\min \left(n_{t}, n_{r}\right)>n_{e}$.

$n_{t} \geq\left(T+n_{e}\right) / 2$. In Fig. 1(b), we show the s.d.o.f. versus coherence time $T$ for the case where $\min \left(n_{t}, n_{r}\right)>n_{e}$. For $T \geq$ $2 \min \left(n_{t}, n_{r}\right)$, the s.d.o.f. is given by Theorem 1 and is shown to approach that of the case with perfect CSIR as $T$ increases. This is due to the fact that, when coherence time is sufficiently large, the impact due to lack of CSI can be neglected. Similarly, when $T<2 \min \left(n_{t}, n_{r}\right)$, the achievable s.d.o.f. in Lemma 4 is plotted instead.

\section{PROOF OF LEMMA 1}

To prove Lemma 1, we will in fact prove the following stronger result for this case:

$$
C_{S} \leq\left[n_{e} \log \left(1+\frac{P}{\sigma_{r}^{2}}\right)-n_{e} \log \left(1+\frac{P}{\sigma_{e}^{2}}\right)\right]^{+} .
$$

To derive the upper bound (11) on the secrecy capacity, we first note that for a fixed $n_{e}$, the secrecy capacity of the MIMO wiretap channel with $n_{r}=n_{e}$ is always greater than or equal to that of the case with $n_{r}<n_{e}$. Hence, it suffices to upper bound the secrecy capacity of the system with $n_{r}=n_{e}$, which we will call the enhanced wiretap channel.
For the enhanced wiretap channel, if $\sigma_{r}^{2} \geq \sigma_{e}^{2}$, it is clear that the legitimate receiver is stochastically degraded with respect to the eavesdropper. Hence, the secrecy capacity in this case is zero. However, if $\sigma_{r}^{2}<\sigma_{e}^{2}$, using the two conditions $n_{r}=n_{e}$ and $\sigma_{r}^{2}<\sigma_{e}^{2}$, we can construct a physically degraded wiretap channel whose marginal distributions are identical to those of (2) and (3). The received signals of the equivalent degraded wiretap channel are

$$
\begin{aligned}
\mathbf{Y} & =\mathbf{H X}+\mathbf{N}_{r} \\
\mathbf{Z}^{\prime} & =\mathbf{H X}+\mathbf{N}_{r}+\mathbf{N}_{e}^{\prime}=\mathbf{Y}+\mathbf{N}_{e}^{\prime}
\end{aligned}
$$

where the entries of $\mathbf{N}_{e}^{\prime} \in \mathcal{C}^{n_{e} \times T}$ are i.i.d. Gaussian with zeromean and variance $\sigma_{e}^{2}-\sigma_{r}^{2}$, and $\mathbf{N}_{e}^{\prime}$ is independent of $\mathbf{X}, \mathbf{H}$, and $\mathbf{N}_{r}$. Since the secrecy capacity depends only on the conditional marginal probabilities $p(\mathbf{Y} \mid \mathbf{X})$ and $p(\mathbf{Z} \mid \mathbf{X})$, and $\mathbf{H}$ and $\mathbf{G}$ are statistically the same, the physically degraded channel in (12) and (13) has the same secrecy capacity as the original stochastically degraded channel in (2) and (3). Due to the degradedness of the equivalent model in (12) and (13), we know, from [2], [3], that $V=\mathbf{X}$ is optimal (i.e., no channel prefixing is needed) and, thus, the secrecy capacity of the equivalent degraded wiretap channel is

$$
C_{s}=\frac{1}{T} \max _{p_{\mathbf{X}} \in S_{p_{\mathbf{X}}}} I(\mathbf{X} ; \mathbf{Y})-I\left(\mathbf{X} ; \mathbf{Z}^{\prime}\right)
$$

where $S_{p_{\mathbf{X}}}$ denotes the set of all input distributions which satisfy the power constraint in (1).

To derive an upper bound we first rewrite (14) as

$$
T \cdot C_{s}=\max _{p_{\mathbf{X}} \in S_{p_{\mathbf{X}}}} h(\mathbf{Y})-h\left(\mathbf{Z}^{\prime}\right)-h(\mathbf{Y} \mid \mathbf{X})+h\left(\mathbf{Z}^{\prime} \mid \mathbf{X}\right) .
$$

Now we note that if $n_{r}=n_{e}$ and $\sigma_{r}^{2}<\sigma_{e}^{2}$, we have the following inequality for the wiretap channel in (12) and (13),

$$
h(\mathbf{Y} \mid \mathbf{X})-h\left(\mathbf{Z}^{\prime} \mid \mathbf{X}\right) \geq h(\mathbf{Y} \mid \mathbf{X}, \mathbf{H})-h\left(\mathbf{Z}^{\prime} \mid \mathbf{X}, \mathbf{H}\right) .
$$

This is a vector generalization of [20, eqn. (12)], and can be proved by observing that (16) holds if and only if

$$
I(\mathbf{Y} ; \mathbf{H} \mid \mathbf{X}) \geq I\left(\mathbf{Z}^{\prime} ; \mathbf{H} \mid \mathbf{X}\right)
$$

which is true since,

$$
\begin{aligned}
I(\mathbf{Y} ; \mathbf{H} \mid \mathbf{X}=\tilde{\mathbf{X}}) & =n_{r} \log \left|\mathbf{I}_{T}+\frac{\tilde{\mathbf{X}}^{\dagger} \tilde{\mathbf{X}}}{\sigma_{r}^{2}}\right| \\
& \geq n_{e} \log \left|\mathbf{I}_{T}+\frac{\tilde{\mathbf{X}}^{\dagger} \tilde{\mathbf{X}}}{\sigma_{e}^{2}}\right| \\
& =I\left(\mathbf{Z}^{\prime} ; \mathbf{H} \mid \mathbf{X}=\tilde{\mathbf{X}}\right),
\end{aligned}
$$

where $\tilde{\mathbf{X}}$ denotes a realization of the random matrix $\mathbf{X}$. In deriving (18), we used the fact that $n_{r}=n_{e}, \sigma_{r}^{2}<\sigma_{e}^{2}$ and that given $\mathbf{X}, \mathbf{Y}$ and $\mathbf{H}$ are jointly Gaussian and so are $\mathbf{Z}$ and $\mathbf{H}$. If $\mathbf{Y}_{i}$ denotes the $i$ th row of $\mathbf{Y}$, then $\mathbf{Y}_{i}$ is a Gaussian vector independent of $\mathbf{Y}_{j}$, for all $i \neq j$, and the covariance matrix of $\mathbf{Y}_{i}$ is $\tilde{\mathbf{X}}^{\dagger} \tilde{\mathbf{X}}+\sigma_{r}^{2} \mathbf{I}_{T}$, for all $i$. 
Using (16) and (13) in (15), we obtain

$$
\begin{aligned}
& T \cdot C_{s} \leq \max _{p_{\mathbf{X}} \in S_{p_{\mathbf{X}}}} h(\mathbf{Y})-h\left(\mathbf{Y}+\mathbf{N}_{e}^{\prime}\right) \\
& -h(\mathbf{Y} \mid \mathbf{X}, \mathbf{H})+h\left(\mathbf{Z}^{\prime} \mid \mathbf{X}, \mathbf{H}\right) \\
& =\max _{p_{\mathbf{X}} \in S_{p_{\mathbf{X}}}} h(\mathbf{Y})-h\left(\mathbf{Y}+\mathbf{N}_{e}^{\prime}\right)+n_{e} T \log \left(\frac{\sigma_{e}^{2}}{\sigma_{r}^{2}}\right) \\
& \leq \max _{p_{\mathbf{X}} \in S_{p_{\mathbf{X}}}} h(\mathbf{Y})-n_{e} T \log \left(e^{\frac{1}{n_{e} T} h(\mathbf{Y})}+e^{\frac{1}{n_{e} T} h\left(\mathbf{N}_{e}^{\prime}\right)}\right) \\
& +n_{e} T \log \left(\frac{\sigma_{e}^{2}}{\sigma_{r}^{2}}\right) \\
& \leq h\left(\mathbf{Y}_{G}\right)-n_{e} T \log \left(e^{\frac{1}{n_{e} T} h\left(\mathbf{Y}_{G}\right)}+e^{\frac{1}{n_{e} T} h\left(\mathbf{N}_{e}^{\prime}\right)}\right) \\
& +n_{e} T \log \left(\frac{\sigma_{e}^{2}}{\sigma_{r}^{2}}\right) \\
& =T n_{e} \log \left(1+\frac{P}{\sigma_{r}^{2}}\right)-T n_{e} \log \left(1+\frac{P}{\sigma_{e}^{2}}\right),
\end{aligned}
$$

where (21) follows from the entropy power inequality [23], and (22) follows from the fact that the right-hand-side of (21) is monotonically increasing in $h(\mathbf{Y})$, and that for a fixed total power constraint $\mathbb{E}\left[\operatorname{tr}\left(\mathbf{Y} \mathbf{Y}^{\dagger}\right)\right] \leq\left(P+\sigma_{r}^{2}\right) n_{e} T$, a Gaussian matrix $\mathbf{Y}_{G} \in \mathrm{C}^{n_{e} \times T}$ with entries that are i.i.d. Gaussian with zero-mean and variance $P+\sigma_{r}^{2}$ maximizes the differential entropy. This gives us the desired result in (11), completing the proof of Lemma 1.

\section{Proof of Lemma 2}

The proof of Lemma 2 is a bit more involved than that of Lemma 1. For clarity, we outline the steps of the proof here and leave the details to Appendix A. We first prove the converse and then provide a scheme that achieves the s.d.o.f. upper bound.

\section{A. Converse Proof of Lemma 2}

To find an upper bound for the s.d.o.f. $D_{s}$, we only need to consider the case where $\sigma_{r}^{2}<\sigma_{e}^{2}$, since, with all other channel parameters remaining the same, the wiretap channel in (2) and (3) with $\sigma_{r}^{2}<\sigma_{e}^{2}$ yields a larger secrecy capacity than that with $\sigma_{r}^{2} \geq \sigma_{e}^{2}$. Under the assumption $\sigma_{r}^{2}<\sigma_{e}^{2}$, we can once again construct a degraded equivalent channel (as we did in (12) and (13) for $n_{r}=n_{e}$ ), without changing $C_{s}$ by selecting $n_{e}$ row vectors from $n_{r}$ rows of the legitimate channel matrix $\mathbf{H}$ to form a statistically marginally identical eavesdropper channel. For any fixed partition $\mathrm{p}_{1} \cup \mathrm{p}_{2}=\left\{1, \ldots, n_{r}\right\}$ where $\left|\mathrm{p}_{1}\right|=n_{e}$ and $\mathrm{p}_{2}=\left\{1, \ldots, n_{r}\right\} \backslash \mathrm{p}_{1}$, we construct a degraded equivalent channel for (2) and (3) as follows:

$$
\begin{aligned}
\mathbf{Y} & =\mathbf{H X}+\mathbf{N}_{r}, \\
\mathbf{Z}_{\mathrm{p}_{1}} & =\mathbf{H}_{\mathrm{p}_{1}} \mathbf{X}+\mathbf{N}_{r, \mathrm{p}_{1}}+\mathbf{N}_{e}^{\prime}=\mathbf{Y}_{\mathrm{p}_{1}}+\mathbf{N}_{e}^{\prime},
\end{aligned}
$$

where $\mathbf{H}_{\mathrm{p}_{1}}, \mathbf{N}_{r, \mathrm{p}_{1}}$, and $\mathbf{Y}_{\mathrm{p}_{1}}$ denote the collection of row vectors with indices belonging to $\mathrm{p}_{1}$ from $\mathbf{H}, \mathbf{N}_{r}$, and $\mathbf{Y}$, respectively, and $\mathbf{Z}_{\mathrm{p}_{1}}$ denotes the equivalent eavesdropper's received signal constructed from $\mathbf{Y}_{\mathrm{p}_{1}}$. For any partition $\left(\mathrm{p}_{1}, \mathrm{p}_{2}\right)$, as in the proof of Lemma 1, the secrecy capacity of the degraded wiretap channel in (24) and (25) is

$$
C_{s}=\frac{1}{T} \max _{p_{\mathbf{X}} \in S_{p_{\mathbf{X}}}} I(\mathbf{X} ; \mathbf{Y})-I\left(\mathbf{X} ; \mathbf{Z}_{\mathrm{p}_{1}}\right) .
$$

From above, the optimization problem in (4) is transformed to a simpler problem which needs to be optimized only with respect to $\mathbf{X}$ as in (26). However, it is still hard to find the optimal input distribution $p_{\mathbf{X}}$. Instead, we characterize the optimal input structure with respect to (26) for the equivalent degraded channel given in (24) and (25). This helps us restrict possible inputdistributions and simplifies the problem. Interestingly, we show in the sequel that, due to the degradedness of the equivalent wiretap channel in (24) and (25) and the concavity of the secrecy rate in the input distribution for degraded channels [20], the optimal input structure in (26) is the same as the optimal input structure in the channel without secrecy constraints in [21].

Recall that a random matrix $\mathbf{M} \in \mathrm{C}^{N \times T}$ where $T \geq N$ is isotropically distributed (i.d.) if $p(\mathbf{M})=p(\mathbf{M U})$, for all deterministic $T \times T$ unitary matrices $\mathbf{U}$. The optimal input structure for the equivalent degraded wiretap channel in (24) and (25) is characterized in the following lemma.

Lemma 5: When $n_{r}>n_{e}$ and $\sigma_{r}^{2}<\sigma_{e}^{2}$, for the equivalent channel in (24) and (25), the optimal input distribution that maximizes $C_{s}$ in (26) has the structure

$$
\mathbf{X}=\boldsymbol{\Lambda} \Theta
$$

if $T \geq n_{t}$, where $\boldsymbol{\Lambda}$ is an $n_{t} \times T$ diagonal random matrix with real and non-negative diagonal elements, and $\Theta$ is a $T \times T$ i.d. unitary matrix which is independent of $\boldsymbol{\Lambda}$.

We provide a proof for Lemma 5 in Appendix A.

Although we cannot completely characterize the optimal $\mathbf{X}$, the result in Lemma 5 suffices to derive a useful upper bound for $D_{s}$. We can rewrite the secrecy capacity given in (26) and upper bound it as

$$
\begin{aligned}
T \cdot C_{s}= & \max _{p_{\mathbf{X}} \in S_{p_{\mathbf{X}}}^{*}} I(\mathbf{X} ; \mathbf{Y})-I\left(\mathbf{X} ; \mathbf{Z}_{\mathrm{p}_{1}}\right) \\
= & \max _{p_{\mathbf{X}} \in S_{p_{\mathbf{X}}}^{*}} h\left(\mathbf{Y}_{\mathrm{p}_{1}}\right)+h\left(\mathbf{Y}_{\mathrm{p}_{2}} \mid \mathbf{Y}_{\mathrm{p}_{1}}\right)-h(\mathbf{Y} \mid \mathbf{X}) \\
& -h\left(\mathbf{Y}_{\mathrm{p}_{1}}+\mathbf{N}_{e}^{\prime}\right)+h\left(\mathbf{Z}_{\mathrm{p}_{1}} \mid \mathbf{X}\right) \\
\leq & \max _{p_{\mathbf{X}} \in S_{p_{\mathbf{X}}}^{*}} h\left(\mathbf{Y}_{\mathrm{p}_{2}} \mid \mathbf{Y}_{\mathrm{p}_{1}}\right)-h(\mathbf{Y} \mid \mathbf{X})+h\left(\mathbf{Z}_{\mathrm{p}_{1}} \mid \mathbf{X}\right),
\end{aligned}
$$

where $S_{p_{\mathbf{X}}}^{*}$ in (28) denotes the set of all input distributions having the optimal structure described in Lemma 5 and satisfying the power constraint in (1), matrix $\mathbf{Y}_{\mathrm{p}_{2}}$ in (29) is the collection of row vectors of $\mathbf{Y}$ with indices belonging to $\mathrm{p}_{2}=\left\{1, \ldots, n_{r}\right\} \backslash$ $\mathrm{p}_{1}$, and the inequality (30) follows from $h\left(\mathbf{Y}_{\mathrm{p}_{1}}\right) \leq h\left(\mathbf{Y}_{\mathrm{p}_{1}}+\mathbf{N}_{e}^{\prime}\right)$.

Now continuing from (30), we derive the desired upper bound in three steps.

Step 1: We derive an upper bound for $h\left(\mathbf{Y}_{\mathrm{p}_{2}} \mid \mathbf{Y}_{\mathrm{p}_{1}}\right)$ in terms of $h(\mathbf{Y})$ so that we can focus only on $h(\mathbf{Y})$ later. This upper bound can be derived by using the following lemma.

Lemma 6: Given an $m \times T$ random matrix $\mathbf{M}$ with differential entropy $h(\mathbf{M})$, for all $n \in\{1, \ldots, m\}$, there must exist 
a partition $\left(\mathrm{p}_{1}, \mathrm{p}_{2}\right)$ where $\mathrm{p}_{1} \cup \mathrm{p}_{2}=\{1, \ldots, m\},\left|\mathrm{p}_{1}\right|=n$, and $\left|\mathrm{p}_{2}\right|=m-n$ such that

$$
h\left(\mathbf{M}_{\mathrm{p}_{2}} \mid \mathbf{M}_{\mathrm{p}_{1}}\right) \leq \frac{m-n}{m} h(\mathbf{M}),
$$

where $\mathbf{M}_{\mathrm{p}_{1}}$ and $\mathbf{M}_{\mathrm{p}_{2}}$ denote the collection of row vectors of $\mathbf{M}$ with indices belonging to $\mathrm{p}_{1}$ and $\mathrm{p}_{2}$, respectively.

We provide a proof for Lemma 6 in Appendix B.

Now, from Lemma 6 and (30), we have

$$
T \cdot C_{s} \leq \max _{p_{\mathbf{X}} \in S_{p_{\mathbf{X}}^{*}}^{*}} \frac{n_{r}-n_{e}}{n_{r}} h(\mathbf{Y})-h(\mathbf{Y} \mid \mathbf{X})+h\left(\mathbf{Z}_{\mathrm{p}_{1}} \mid \mathbf{X}\right),
$$

which follows from the fact that, for any partition $\left(\mathrm{p}_{1}, \mathrm{p}_{2}\right),(30)$ is a valid upper bound. A similar inequality for (31) has been derived in [17] under the entropy symmetric condition which is not required in Lemma 6. However, it is necessary to note that the result in [17] is also applicable here since our problem coincidentally satisfies the entropy symmetric condition as well.

Step 2: We derive an upper bound for $h(\mathbf{Y})$ in (32), as given in the following lemma.

Lemma 7: With the distribution of the channel input $\mathbf{X}$ satisfying the optimal structure in Lemma 5, the corresponding differential entropy of the legitimate receiver signal $\mathbf{Y}$ in (12) can be upper bounded as

$$
\max _{p \mathbf{X} \in S_{P_{\mathbf{X}}}^{*}} h(\mathbf{Y}) \leq n_{r}^{2} \log P+\left(T-n_{r}\right) \mathbb{E}\left[\log \operatorname{det} \mathbf{Y} \mathbf{Y}^{\dagger}\right]+o(\log P),
$$

where $\lim _{P \rightarrow \infty} o(\log P) / \log P=0$.

We provide a proof for Lemma 7 in Appendix C.

Note that given the input signal $\mathbf{X}$, each row vector of $\mathbf{Y}$ and $\mathbf{Z}_{\mathrm{p}_{1}}$ are i.i.d. Gaussian vectors, under the optimal input structure imposed by Lemma 5 , the conditional differential entropy $h(\mathbf{Y} \mid \mathbf{X})$ and $h\left(\mathbf{Z}_{\mathrm{p}_{1}} \mid \mathbf{X}\right)$ in (32) can be explicitly computed as

$$
\begin{aligned}
h(\mathbf{Y} \mid \mathbf{X})= & n_{r} \sum_{i=1}^{n_{t}} \mathbb{E}\left[\log \pi e\left(\left\|\mathbf{X}_{i}\right\|^{2}+\sigma_{r}^{2}\right)\right] \\
& +n_{r}\left(T-n_{t}\right) \log \pi e \sigma_{r}^{2}, \\
h\left(\mathbf{Z}_{\mathrm{p}_{1}} \mid \mathbf{X}\right)= & n_{e} \sum_{i=1}^{n_{t}} \mathbb{E}\left[\log \pi e\left(\left\|\mathbf{X}_{i}\right\|^{2}+\sigma_{e}^{2}\right)\right] \\
& +n_{e}\left(T-n_{t}\right) \log \pi e \sigma_{e}^{2},
\end{aligned}
$$

where $\mathbf{X}_{i}$ is the $i$ th row of the given input signal $\mathbf{X}$.

Now, by Lemma 7 and (32)-(35), we can further upper bound the secrecy capacity in (32) as

$$
\begin{aligned}
T \cdot C_{s} \leq \max _{p_{\mathbf{X}} \in S_{p_{\mathbf{X}}^{*}}^{*}}\{ & \frac{n_{r}-n_{e}}{n_{r}}\left(T-n_{r}\right) \mathbb{E}\left[\log \operatorname{det} \mathbf{Y} \mathbf{Y}^{\dagger}\right] \\
& -\left(n_{r}-n_{e}\right) \sum_{i=1}^{n_{t}} \mathbb{E}\left[\log \left(\left\|\mathbf{X}_{i}\right\|^{2}+\sigma_{r}^{2}\right)\right] \\
& +n_{e} \sum_{i=1}^{n_{t}} \mathbb{E}\left[\log \left(\frac{\left\|\mathbf{X}_{i}\right\|^{2}+\sigma_{e}^{2}}{\left\|\mathbf{X}_{i}\right\|^{2}+\sigma_{r}^{2}}\right)\right] \\
& \left.+\left(n_{r}-n_{e}\right) n_{r} \log P+o(\log P)\right\} .
\end{aligned}
$$

Furthermore, by using the fact that $\log (1+x) \leq x$, it follows that

$$
\mathbb{E}\left[\log \left(\frac{\left\|\mathbf{X}_{i}\right\|^{2}+\sigma_{e}^{2}}{\left\|\mathbf{X}_{i}\right\|^{2}+\sigma_{r}^{2}}\right)\right] \leq \mathbb{E}\left[\frac{\sigma_{e}^{2}-\sigma_{r}^{2}}{\left\|\mathbf{X}_{i}\right\|^{2}+\sigma_{r}^{2}}\right] \leq \frac{\sigma_{e}^{2}-\sigma_{r}^{2}}{\sigma_{r}^{2}},
$$

where the right-hand-side of (37) is a constant independent of $P$. Therefore, by (36) and (37), we can upper bound the secrecy capacity $T \cdot C_{S}$ as

$$
\begin{aligned}
T \cdot C_{s} \leq \max _{p_{\mathbf{X}} \in S_{p_{\mathbf{X}}}^{*}} & \left\{( n _ { r } - n _ { e } ) \left(\frac{\left(T-2 n_{r}\right)}{n_{r}} \mathbb{E}\left[\log \operatorname{det} \mathbf{Y} \mathbf{Y}^{\dagger}\right]\right.\right. \\
& \left.+\mathbb{E}\left[\log \operatorname{det} \mathbf{Y} \mathbf{Y}^{\dagger}\right]-\sum_{i=1}^{n_{t}} \mathbb{E}\left[\log \left(\left\|\mathbf{X}_{i}\right\|^{2}+\sigma_{r}^{2}\right)\right]\right) \\
& \left.+\left(n_{r}-n_{e}\right) n_{r} \log P+o(\log P)\right\}
\end{aligned}
$$

By the assumptions $T \geq 2 n_{r}$ and $n_{r}>n_{e}$, we obtain a further upper bound for (38) by developing upper bounds separately for $\mathbb{E}\left[\log \operatorname{det} \mathbf{Y} \mathbf{Y}^{\dagger}\right]$ and $\mathbb{E}\left[\log \operatorname{det} \mathbf{Y} \mathbf{Y}^{\dagger}\right]-\sum_{i=1}^{n_{t}} \mathbb{E}\left[\log \left(\left\|\mathbf{X}_{i}\right\|^{2}+\boldsymbol{\sigma}_{r}^{2}\right)\right]$, respectively. This is the task of step 3 .

Step 3: We derive upper bounds for the two terms $\mathbb{E}\left[\log \operatorname{det} \mathbf{Y} \mathbf{Y}^{\dagger}\right]$ and $\mathbb{E}\left[\log \operatorname{det} \mathbf{Y} \mathbf{Y}^{\dagger}\right]-\sum_{i=1}^{n_{t}} \mathbb{E}\left[\log \left(\left\|\mathbf{X}_{i}\right\|^{2}+\sigma_{r}^{2}\right)\right]$ in (38) separately using the following two lemmas.

Lemma 8: With the distribution of the channel input $\mathbf{X}$ satisfying the optimal structure in Lemma 5, and with $n_{t} \geq n_{r}$, the legitimate received signal $\mathbf{Y}$ in (12) satisfies

$$
\max _{p_{\mathbf{X}} \in S_{p_{\mathbf{X}}}^{*}} \mathbb{E}\left[\log \operatorname{det} \mathbf{Y} \mathbf{Y}^{\dagger}\right] \leq n_{r} \log P+o(\log P),
$$

where $\lim _{P \rightarrow \infty} o(\log P) / \log P=0$.

Lemma 9: With the distribution of the channel input $\mathbf{X}$ satisfying the optimal structure in Lemma 5 , and with $n_{t} \geq n_{r}$, the legitimate received signal $\mathbf{Y}$ in (12) satisfies

$$
\max _{p_{\mathbf{X}} \in S_{P_{\mathbf{X}}}^{*}} \mathbb{E}\left[\log \operatorname{det} \mathbf{Y} \mathbf{Y}^{\dagger}\right]-\sum_{i=1}^{n_{t}} \mathbb{E}\left[\log \left(\left\|\mathbf{X}_{i}\right\|^{2}+\sigma_{r}^{2}\right)\right] \leq k,
$$

where $k$ is a constant which is independent of $P$.

We provide proofs for Lemmas 8 and 9 in Appendices D and E, respectively. It should be mentioned that here we focus on the setting where $n_{t} \geq n_{r}$, and the random channel matrix $\mathbf{H}$ is not full column rank. Thus, the results of [22] cannot be directly applied to prove Lemmas 8 and 9 . More discussion on this can be found at the end of Appendix E. In addition, in [22], where the conventional MIMO channel with no eavesdroppers was examined, a key step in proving the converse of the d.o.f. was the upper-bounding of the mutual information $I(\mathbf{X} ; \mathbf{Y})$. However, when proving the s.d.o.f. of wiretap channels, one instead needs to derive upper bounds for the difference in mutual information $I(\mathbf{X} ; \mathbf{Y})-I(\mathbf{X} ; \mathbf{Z})$. The results in [22] do not apply in this case and, thus, new upper-bounding techniques are developed here.

Finally, using Lemmas 8 and 9 in (38), we obtain the desired upper bound on the s.d.o.f. as

$$
D_{s} \leq\left(n_{r}-n_{e}\right)\left(\frac{T-n_{r}}{T}\right),
$$


which completes the converse part of Lemma 2.

\section{B. Achievability Proof of Lemma 2}

Here, we show that a constant norm channel input [21], [22] transmitted on $n_{r}$ antennas can achieve the s.d.o.f. upper bound given in (41). Specifically, let the channel input $\mathbf{X}_{c}$ be constant norm over $n_{r}$ transmitter antennas and zero over the rest of the $n_{t}-n_{r}$ antennas, i.e.,

$$
\mathbf{X}_{c}=\left[\begin{array}{cc}
\sqrt{\frac{P T}{n_{r}}} \mathbf{I}_{n_{r}} & \mathbf{0} \\
\mathbf{0} & \mathbf{0}
\end{array}\right] \boldsymbol{\Theta},
$$

where $\boldsymbol{\Theta}$ is an $T \times T$ i.d. unitary matrix and $\mathbf{I}_{n_{r}}$ denotes the identity matrix with $n_{r}$ dimension. We can lower bound the achievable secrecy rate $R_{s}$ as follows:

$$
\begin{aligned}
T \cdot R_{S}= & I\left(\mathbf{X}_{c} ; \mathbf{Y}\right)-I\left(\mathbf{X}_{c} ; \mathbf{Z}\right) \\
= & h(\mathbf{Y})-h(\mathbf{Z})-h\left(\mathbf{Y} \mid \mathbf{X}_{c}\right)+h\left(\mathbf{Z} \mid \mathbf{X}_{c}\right) \\
= & h(\mathbf{Y})-h(\mathbf{Z}) \\
& -n_{r} \sum_{i=1}^{n_{r}} \log \left(\frac{P T}{n_{r}}+\sigma_{r}^{2}\right)-n_{r}\left(T-n_{t}\right) \log \pi e \sigma_{r}^{2} \\
& +n_{e} \sum_{i=1}^{n_{r}} \log \left(\frac{P T}{n_{r}}+\sigma_{e}^{2}\right)+n_{e}\left(T-n_{t}\right) \log \pi e \sigma_{e}^{2} \\
\geq & h(\mathbf{Y})-h(\mathbf{Z})-\left(n_{r}-n_{e}\right) n_{r} \log P+o(\log P),
\end{aligned}
$$

where (45) follows by applying (42) into (34) and (35), respectively.

Since $\mathbb{E}\left[\operatorname{tr}\left(\mathbf{Z Z} \mathbf{Z}^{\dagger}\right)\right] \leq\left(P+\sigma_{e}^{2}\right) n_{e} T$, the differential entropy $h(\mathbf{Z})$ of $\mathbf{Z}$ can be upper bounded by the differential entropy of an i.i.d. Gaussian matrix as

$$
h(\mathbf{Z}) \leq n_{e} T \log \left(\pi e\left(P+\sigma_{e}^{2}\right)\right)=n_{e} T \log P+o(\log P),
$$

and, the differential entropy $h(\mathbf{Y})$ can be lower bounded as

$$
\begin{aligned}
h(\mathbf{Y}) \geq & h\left(\mathbf{H} \mathbf{X}_{c}\right) \\
= & h\left(\mathbf{C}_{\mathbf{H}}\right)+\log \left|G\left(n_{r}, T\right)\right| \\
& +\left(T-n_{r}\right) \mathbb{E}\left[\log \operatorname{det} \mathbf{H} \mathbf{X}_{c} \mathbf{X}_{c}^{\dagger} \mathbf{H}^{\dagger}\right] \\
= & h\left(\sqrt{\frac{P T}{n_{r}}} \mathbf{H}_{a}\right)+\log \left|G\left(n_{r}, T\right)\right| \\
& +\left(T-n_{r}\right) \mathbb{E}\left[\log \operatorname{det} \frac{P T}{n_{r}} \mathbf{H}_{a} \mathbf{H}_{a}^{\dagger}\right] \\
= & n_{r}^{2} \log \pi e \frac{P T}{n_{r}}+\log \left|G\left(n_{r}, T\right)\right|+\left(T-n_{r}\right) \log \left(\frac{P T}{n_{r}}\right)^{n_{r}} \\
& +\left(T-n_{r}\right) \mathbb{E}\left[\log \operatorname{det} \mathbf{H}_{a} \mathbf{H}_{a}^{\dagger}\right] \\
= & n_{r} T \log P+o(\log P),
\end{aligned}
$$

where $\mathbf{H}_{a}$ denotes the collection of the first $n_{r}$ columns of matrix $\mathbf{H}$ and $\left|G\left(n_{r}, T\right)\right|$ is the volume of the Grassmann Manifold $G\left(n_{r}, T\right)$ (c.f. Appendix C), which is a finite constant. Note that (49) is obtained by applying Lemma 15 in Appendix C.
A similar derivation, for the case where $n_{t}=n_{r}$, can be found in the proof of Lemma 15 in [22]. Therefore, from (46), (47), and (52), we have the following lower bound on the secrecy rate

$$
T \cdot R_{s} \geq\left(n_{r}-n_{e}\right)\left(T-n_{r}\right) \log P+o(\log P),
$$

which implies that

$$
D_{s} \geq\left(n_{r}-n_{e}\right)\left(\frac{T-n_{r}}{T}\right) .
$$

Together with the upper bound in (41), we conclude that, the exact s.d.o.f. for the case $n_{t} \geq n_{r}>n_{e}$ and $T \geq 2 n_{r}$, is

$$
D_{s}=\left(n_{r}-n_{e}\right)\left(\frac{T-n_{r}}{T}\right),
$$

which completes the proof of Lemma 2.

As a final remark, we note that when $n_{t} \geq n_{r}$ and $T \geq 2 n_{r}$, we can use only $n_{r}$ transmitter antennas to achieve the optimal s.d.o.f in (55). Having more than $n_{r}$ transmit antennas gives us no improvements, at least, as far as the s.d.o.f. is concerned.

\section{Proof of Lemma 3}

The proof is based on the key observation that, when $n_{t}<n_{r}$, the receiver can use only $n_{t}$ of its antennas without losing any s.d.o.f. That is, for a fixed $n_{t}$, the s.d.o.f. in the case where $n_{t}<$ $n_{r}$ is, in fact, equal to the s.d.o.f. in the case with $n_{r}=n_{t}$. This fact is shown in the following lemma.

Lemma 10: For the MIMO legitimate channel (2), if $n_{t}<n_{r}$, for any input signal $\mathbf{X}$ satisfying the power constraint in (1), we have

$$
I(\mathbf{X} ; \mathbf{Y})-I\left(\mathbf{X} ; \mathbf{Y}_{n_{t}}\right) \leq o(\log P)
$$

where $\mathbf{Y}_{n_{t}}$ denotes the collection of arbitrary $n_{t}$ row vectors of the received signal matrix $\mathbf{Y}$.

We provide a proof for Lemma 10 in Appendix F.

To derive an upper bound for the s.d.o.f., we first focus on the case $\sigma_{r}^{2}<\sigma_{e}^{2}$. When $n_{r}>n_{e}$ and $\sigma_{r}^{2}<\sigma_{e}^{2}$, we can construct the same equivalent degraded channel in (24) and (25), in which case, the secrecy capacity can be written as in (26). The only difference here is that now the number of transmitter antennas is less than the number of legitimate receiver antennas, i.e., $n_{t}<$ $n_{r}$. If we denote $C_{s}^{n_{t}<n_{r}}$ as the secrecy capacity of the wiretap channel in (2) and (3) with $\sigma_{r}^{2}<\sigma_{e}^{2}$ and $n_{t}<n_{r}$, and $\mathbf{X}^{*}$ as the corresponding optimal input, we have

$$
\begin{aligned}
T \cdot C_{s}^{n_{t}<n_{r}}= & I\left(\mathbf{X}^{*} ; \mathbf{Y}\right)-I\left(\mathbf{X}^{*} ; \mathbf{Z}\right) \\
& \leq I\left(\mathbf{X}^{*} ; \mathbf{Y}_{n_{t}}\right)-I\left(\mathbf{X}^{*} ; \mathbf{Z}\right)+o(\log P) \\
& \leq \max _{p_{\mathbf{X}} \in S_{p_{\mathbf{X}}}}\left[I\left(\mathbf{X} ; \mathbf{Y}_{n_{t}}\right)-I(\mathbf{X} ; \mathbf{Z})\right]+o(\log P) \\
& =T \cdot C_{s}^{n_{t}=n_{r}}+o(\log P)
\end{aligned}
$$

where (58) follows from Lemma 10, and $C_{s}^{n_{t}=n_{r}}$ in (60) is the secrecy capacity of the wiretap channel in (2) and (3) with smaller $n_{r}$ as $n_{t}=n_{r}$. It is worthwhile to note that, in [22], a 
result similar to (56) but with more restrictions was given as

$$
\max _{p_{\mathbf{X}} \in S_{p_{\mathbf{X}}}} I(\mathbf{X} ; \mathbf{Y})-\max _{p_{\mathbf{X}} \in S_{p_{\mathbf{X}}}} I\left(\mathbf{X} ; \mathbf{Y}_{n_{t}}\right) \leq o(\log P) .
$$

The inequality in (61) is useful to prove the results in [22] for conventional MIMO channels, but is not sufficient for the proofs in MIMO wiretap channels. This is because, in (61), an upper bound was obtained when the input distribution is the one that maximizes $I(\mathbf{X} ; \mathbf{Y})$. However, to obtain the inequality in (59), the input distribution must be one that maximizes the difference $I\left(\mathbf{X} ; \mathbf{Y}_{n_{t}}\right)-I(\mathbf{X} ; \mathbf{Z})$ instead. Thus, the more general result in Lemma 10 is required.

We already know the s.d.o.f. when $n_{r}=n_{t}$ and $T \geq 2 n_{r}$. For $n_{r}=n_{t}>n_{e}$, the s.d.o.f. $D_{s}$ is given by (55) from Lemma 2, and for $n_{r}=n_{t} \leq n_{e}, D_{s}=0$ from Lemma 1 . Thus, when $n_{t}<n_{r}$ and $T \geq 2 n_{t}$, we get the required upper bound as

$$
D_{s} \leq\left(n_{t}-n_{e}\right)^{+}\left(\frac{T-n_{t}}{T}\right) .
$$

Furthermore, in the case where $\sigma_{r}^{2} \geq \sigma_{e}^{2}$, one can upper bound the secrecy capacity by increasing $\sigma_{e}^{2}$ in the eavesdropper's channel. Thus, the upper bound (62) is still valid.

The achievability of the above upper bound follows by using a constant norm channel input over $n_{t}$ transmitter antennas as described in Section V-B. However, at the legitimate receiver, only $n_{t}$ receiver antennas are needed and we can ignore the remaining $\left(n_{r}-n_{t}\right)$ row vectors of the received signal matrix $\mathbf{Y}$ while decoding at high SNR. These matching converse and achievability results complete the proof of Lemma 3 .

\section{SeCure Degrees of FreEdom For Short COHERENCE TIME Systems}

In this section, we provide some insights for the s.d.o.f. of short coherence time systems (i.e., systems with $T<$ $2 \min \left\{n_{t}, n_{r}\right\}$ ). Recall that, when $n_{r} \leq n_{e}$, we know from Lemma 1 that the s.d.o.f. is zero regardless of the coherence time $T$. However, when $n_{r}>n_{e}$, our results in Theorem 1 hold only for the case with sufficiently large coherence time, i.e., for $T \geq 2 \min \left(n_{t}, n_{r}\right)$. To study the s.d.o.f of short coherence time systems, we first consider a special case where $T=1$ (i.e., fast fading channels). We show, in the following lemma, that the s.d.o.f. of the MIMO fast fading wiretap channel is zero regardless of how many antennas the terminals have. This is a generalization of [20] to the case of multiple antennas.

Lemma 11: For the MIMO wiretap channel in (2) and (3), with no CSI at any terminal and $T=1$, we have $D_{s}=0$.

Proof: Here, we focus only on the case where $n_{r}>n_{e}$ since the s.d.o.f. is zero when $n_{r} \leq n_{e}$ (c.f., Lemma 1). Specifically, let us first consider the case where $n_{r}>n_{e}$ and $\sigma_{r}^{2} \leq \sigma_{e}^{2}$. In this case, the MIMO wiretap channel can be converted to an equivalent degraded wiretap channel as in (24) and (25), similar to what was done in Section V, and its secrecy capacity can be written as in (26). Following the result in [21], we note in the following lemma that, when $n_{t} \geq T$, which is always the case when $T=1$, the secrecy capacity of the equivalent degraded wiretap channel can be achieved by using only $n_{t}=T$ transmit antennas.

Lemma 12: Suppose that $n_{t}>T$ and that the $n_{t} \times T$ input signal $\mathbf{X}$ with distribution $p_{\mathbf{X}}$ generates mutual informations $I(\mathbf{X} ; \mathbf{Y})$ and $I\left(\mathbf{X} ; \mathbf{Z}_{\mathrm{p}_{1}}\right)$ on the main and the eavesdropper channels, respectively, described in (24) and (25). Then, there exists an $T \times T$ input signal $\mathbf{X}^{\prime}$, i.e., an input signal that utilizes only $T$ transmit antennas, that generates the same mutual informations, i.e., $I(\mathbf{X} ; \mathbf{Y})=I\left(\mathbf{X}^{\prime} ; \mathbf{Y}\right)$ and $I\left(\mathbf{X} ; \mathbf{Z}_{\mathrm{p}_{1}}\right)=I\left(\mathbf{X}^{\prime} ; \mathbf{Z}_{\mathrm{p}_{1}}\right)$.

This lemma is a straightforward extension of [21, Theorem 1] and, thus, its proof is omitted here. The main idea is that both conditional probability density functions $p(\mathbf{Y} \mid \mathbf{X})$ and $p\left(\mathbf{Z}_{\mathrm{p}_{1}} \mid \mathbf{X}\right)$ depend on $\mathbf{X}$ only through $\mathbf{X}^{\dagger} \mathbf{X}$. Hence, for any $n_{t} \times T$ input matrix $\mathbf{X}$, we can obtain the same mutual informations by using a $T \times T$ input matrix $\mathbf{X}^{\prime}$ such that $\mathbf{X}^{\prime \dagger} \mathbf{X}^{\prime}=\mathbf{X}^{\dagger} \mathbf{X}$.

It follows from Lemma 12 that, when $T=1$, the secrecy capacity is the same as the secrecy capacity with a single transmit antenna only, i.e., $n_{t}=1$. Moreover, by Lemma 10 , we know that having more receive antennas than transmit antennas, i.e., having $n_{r}>n_{t}$, does not improve the s.d.o.f. Thus, when $n_{r}>n_{e}, \sigma_{r}^{2}<\sigma_{e}^{2}$ and $T=1$, the s.d.o.f. of the MIMO wiretap channel is the same as the secrecy capacity of the SISO case, i.e., $n_{t}=n_{r}=1$, which is zero, as shown in [20]. Finally, since the secrecy capacity for the case with $\sigma_{r}^{2} \leq \sigma_{e}^{2}$ is greater than that with $\sigma_{r}^{2}>\sigma_{e}^{2}$, we conclude that the s.d.o.f. of the fast fading wiretap channel, i.e., the case with $T=1$, is zero regardless of the number of antennas at the terminals.

For the general case, the exact s.d.o.f. is unknown. However, as stated in Lemma 4, we can show that, by using constant norm input, the achievable s.d.o.f. can be given by

$$
\left(K-n_{e}\right)^{+}\left(\frac{T-K}{T}\right)
$$

where $K \triangleq \min \left(n_{t}, n_{r},\left(T+n_{e}\right) / 2\right)$. To show this, we first note from Lemma 12 that, when $T<n_{t}$, the secrecy capacity can be achieved by using only $T$ out of the $n_{t}$ transmit antennas. That is, no further improvement in secrecy capacity can be obtained by using all $n_{t}$ antennas. Therefore, in the following, we focus only on the case where $n_{t} \leq T$.

Suppose that the constant norm input is applied over $m \leq$ $\min \left(n_{t}, n_{r}\right)$ transmitter antennas (c.f. in Section V-B) and let the channel input signal be written as

$$
\mathbf{X}=\left[\begin{array}{cc}
\sqrt{\frac{P T}{m}} \mathbf{I}_{m} & \mathbf{0} \\
\mathbf{0} & \mathbf{0}
\end{array}\right] \boldsymbol{\Theta} .
$$

Moreover, let us also assume that only $m$ antennas are used at the legitimate receiver to receive the signal. Then, by following the same arguments as in Section V-B, we can show that the s.d.o.f. achieved by using (64) is given by

$$
\left(m-n_{e}\right)^{+}\left(\frac{T-m}{T}\right) \text {. }
$$

Note that (65) is a quadratic function that increases with $m$ when $n_{e}<m<\left(T+n_{e}\right) / 2$ and reaches its maximum value at the point $m=\left(T+n_{e}\right) / 2$. Thus, together with the condition $m \leq \min \left(n_{t}, n_{r}\right)$, the number of transmit and receive antennas 
that should be used to transmit the constant norm input signal is $m=K$ and the resulting achievable s.d.o.f. is given by (63). However, whether or not (63) is the maximum achievable s.d.o.f. is still an open problem.

\section{CONCLUSION}

We considered the Rayleigh block fading wiretap channel with no a priori CSI at any of the terminals. We constructed a degraded equivalent channel, and determined its secrecy capacity. We determined the exact s.d.o.f. of this channel model, when $T \geq 2 \min \left(n_{t}, n_{r}\right)$, to be $\left(\min \left(n_{t}, n_{r}\right)-n_{e}\right)^{+}(T-$ $\left.\min \left(n_{t}, n_{r}\right)\right) / T$. When $\min \left(n_{t}, n_{r}\right) \leq n_{e}$, the s.d.o.f. is zero no matter how long the coherence time $T$ is; an example of this is the scalar wiretap channel where $n_{t}=n_{r}=n_{e}=1$. When $T=1$, the s.d.o.f. is zero no matter how many antennas the transmitter and the legitimate receiver may have. We showed in this paper that when we have some moderate channel coherence together with multiple antennas at the legitimate entities, we can have non-zero s.d.o.f. The needed condition for this is that the legitimate entities have more antennas than the eavesdropper.

\section{APPENDIX A}

PROOF OF LEMMA 5

We first introduce the following two lemmas which will be useful for the proof of Lemma 5. These lemmas are straightforward extensions of Lemmas 1 and 3 of [21].

Lemma 13: Suppose that the input signal $\mathbf{X}$ with distribution $p_{\mathbf{X}}$ generates mutual informations $I(\mathbf{X} ; \mathbf{Y})$ and $I\left(\mathbf{X} ; \mathbf{Z}_{\mathrm{p}_{1}}\right)$ on the main and the eavesdropper channels described in (24) and (25). For any $m \times m$ deterministic unitary matrix $\mathbf{V}$ and $T \times T$ deterministic unitary matrix $\mathbf{U}$, the input signal $\mathbf{V}^{\dagger} \mathbf{X U}$ generates the same mutual informations, i.e., $I(\mathbf{X} ; \mathbf{Y})=I\left(\mathbf{V}^{\dagger} \mathbf{X U} ; \mathbf{Y}\right)$ and $I\left(\mathbf{X} ; \mathbf{Z}_{\mathrm{p}_{1}}\right)=I\left(\mathbf{V}^{\dagger} \mathbf{X U} ; \mathbf{Z}_{\mathrm{p}_{1}}\right)$.

Lemma 14: Suppose that the input signal $\mathbf{X}$ with singular value decomposition $\mathbf{X}=\boldsymbol{\Psi}^{\dagger} \boldsymbol{\Lambda} \boldsymbol{\Phi}$ generates the mutual informations $I(\mathbf{X} ; \mathbf{Y})$ and $I\left(\mathbf{X} ; \mathbf{Z}_{\mathrm{p}_{1}}\right)$ on the main and the eavesdropper channels described in (24) and (25). Then, the input signal $\mathbf{X}^{\prime}=\mathbf{\Lambda} \boldsymbol{\Phi}$ also generates the same mutual informations, i.e., $I(\mathbf{X} ; \mathbf{Y})=I\left(\mathbf{X}^{\prime} ; \mathbf{Y}\right)$ and $I\left(\mathbf{X} ; \mathbf{Z}_{\mathrm{p}_{1}}\right)=I\left(\mathbf{X}^{\prime} ; \mathbf{Z}_{\mathrm{p}_{1}}\right)$.

Note that, the above lemmas hold separately for $I(\mathbf{X} ; \mathbf{Y})$ and $I\left(\mathbf{X} ; \mathbf{Z}_{\mathrm{p}_{1}}\right)$ irrespective of the degradedness relation, and relies only on the fact that their respective channels, i.e., $p_{\mathbf{Y} \mid \mathbf{X}}$ and $p_{\mathbf{Z}_{\mathrm{p}_{1}} \mid \mathbf{X}}$, are Gaussian. Using the above two lemmas, we will show that, for any input $\mathbf{X}$ with distribution $p_{\mathbf{X}}$, there exists input signal $\mathbf{X}^{*}$, satisfying the structure in (27) in Lemma 5, that achieves a higher secrecy rate for the degraded MIMO wiretap channel given in (24) and (25), i.e.,

$$
\frac{1}{T}\left(I(\mathbf{X} ; \mathbf{Y})-I\left(\mathbf{X} ; \mathbf{Z}_{\mathrm{p}_{1}}\right)\right) \leq \frac{1}{T}\left(I\left(\mathbf{X}^{*} ; \mathbf{Y}\right)-I\left(\mathbf{X}^{*} ; \mathbf{Z}_{\mathrm{p}_{1}}\right)\right) .
$$

To do this, let us define the left-hand-side as the secrecy rate function $R_{s}\left(p_{\mathbf{X}}\right)=\frac{1}{T}\left(I(\mathbf{X} ; \mathbf{Y})-I\left(\mathbf{X} ; \mathbf{Z}_{\mathrm{p}_{1}}\right)\right)$ where $p_{\mathbf{X}}$ denotes the probability distribution of $\mathbf{X}$. Note that $R_{S}$ is a concave function of the input probability distribution [24] since the eavesdropper's channel (25) is degraded with respect to the main channel (24). Then, for any input signal $\mathbf{X}=\boldsymbol{\Psi}^{\dagger} \boldsymbol{\Lambda} \boldsymbol{\Phi}$, we can let $\mathbf{X}^{\prime}=\boldsymbol{\Lambda} \Phi$ and $\mathbf{X}^{*}=\Lambda \boldsymbol{\Phi} \Theta^{\prime}$ with $\Theta^{\prime}$ being a $T \times T$ i.d. unitary matrix which is independent of $\mathbf{X}$ (independent of $(\boldsymbol{\Psi}, \boldsymbol{\Lambda}, \boldsymbol{\Phi}))$, and upper bound the secrecy rate with the input $\mathbf{X}$ as follows

$$
\begin{aligned}
R_{S}\left(p_{\mathbf{X}}\right) & =R_{S}\left(p_{\mathbf{X}^{\prime}}\right) \\
& =R_{S}\left(p_{\mathbf{X}^{*} \mid \boldsymbol{\Theta}^{\prime}=\tilde{\Theta}^{\prime}}\right) \\
& =\int R_{S}\left(p_{\mathbf{X}^{*} \mid \tilde{\Theta}^{\prime}}\right) d F\left(\tilde{\boldsymbol{\Theta}}^{\prime}\right) \\
& \leq R_{S}\left(\int p_{\mathbf{X}^{*} \mid \tilde{\Theta}^{\prime}} d F\left(\tilde{\boldsymbol{\Theta}}^{\prime}\right)\right) \\
& =R_{S}\left(p_{\mathbf{X}^{*}}\right),
\end{aligned}
$$

where (66) follows from Lemma 14 and the equality in (67) follows from Lemma 13 with $\mathbf{V}$ being the identity matrix and $\mathbf{U}$ being the given realization $\Theta^{\prime}$, and (69) follows from Jensen's inequality since $R_{S}$ is concave.

Now let $\boldsymbol{\Theta}=\boldsymbol{\Phi} \Theta^{\prime}$ such that $\mathbf{X}^{*}=\boldsymbol{\Lambda} \boldsymbol{\Phi} \Theta^{\prime}=\boldsymbol{\Lambda} \Theta$. The rest of the proof is to show that $\boldsymbol{\Theta}$ is also an i.d. unitary matrix and is independent of $\boldsymbol{\Lambda}$. First, we have

$$
\begin{aligned}
p_{\boldsymbol{\Theta}}(\tilde{\boldsymbol{\Theta}}) & =\int p_{\boldsymbol{\Theta} \mid \boldsymbol{\Phi}}(\tilde{\boldsymbol{\Theta}} \mid \tilde{\boldsymbol{\Phi}}) d F(\tilde{\boldsymbol{\Phi}}) \\
& =\int p_{\boldsymbol{\Theta}^{\prime} \mid \boldsymbol{\Phi}}\left(\tilde{\boldsymbol{\Phi}}^{-1} \tilde{\boldsymbol{\Theta}} \mid \tilde{\boldsymbol{\Phi}}\right) d F(\tilde{\boldsymbol{\Phi}}) \\
& =\int p_{\boldsymbol{\Theta}^{\prime}}\left(\tilde{\boldsymbol{\Phi}}^{-1} \tilde{\boldsymbol{\Theta}}\right) d F(\tilde{\boldsymbol{\Phi}}) \\
& =\int p_{\Theta^{\prime}}(\tilde{\boldsymbol{\Theta}}) d F(\tilde{\boldsymbol{\Phi}}) \\
& =p_{\boldsymbol{\Theta}^{\prime}}(\tilde{\boldsymbol{\Theta}}),
\end{aligned}
$$

where (73) comes from the fact that $\boldsymbol{\Theta}^{\prime}$ is independent of $(\boldsymbol{\Psi}, \boldsymbol{\Lambda}, \boldsymbol{\Phi})$ and (74) comes from the fact that $\boldsymbol{\Theta}^{\prime}$ is i.d. unitary [21]. Therefore, we show that $\boldsymbol{\Theta}$ has the same distribution as $\boldsymbol{\Theta}^{\prime}$, and then $\Theta$ is also i.d. unitary. For the independence between $\Theta$ and $\boldsymbol{\Lambda}$, we have

$$
\begin{aligned}
& p_{\boldsymbol{\Theta} \mid \boldsymbol{\Lambda}}(\tilde{\boldsymbol{\Theta}} \mid \tilde{\boldsymbol{\Lambda}})=\int p_{\boldsymbol{\Theta} \mid \boldsymbol{\Lambda}, \boldsymbol{\Phi}}(\tilde{\boldsymbol{\Theta}} \mid \tilde{\boldsymbol{\Lambda}}, \tilde{\boldsymbol{\Phi}}) p_{\boldsymbol{\Phi} \mid \boldsymbol{\Lambda}}(\tilde{\boldsymbol{\Phi}} \mid \tilde{\boldsymbol{\Lambda}}) d \tilde{\boldsymbol{\Phi}} \\
& =\int p_{\boldsymbol{\Theta}^{\prime} \mid \boldsymbol{\Lambda}, \boldsymbol{\Phi}}\left(\tilde{\boldsymbol{\Phi}}^{-1} \tilde{\boldsymbol{\Theta}} \mid \tilde{\boldsymbol{\Lambda}}, \tilde{\boldsymbol{\Phi}}\right) p_{\boldsymbol{\Phi} \mid \boldsymbol{\Lambda}}(\tilde{\boldsymbol{\Phi}} \mid \tilde{\boldsymbol{\Lambda}}) d \tilde{\boldsymbol{\Phi}} \\
& =\int p_{\Theta^{\prime}}\left(\tilde{\boldsymbol{\Phi}}^{-1} \tilde{\boldsymbol{\Theta}}\right) p_{\boldsymbol{\Phi} \mid \boldsymbol{\Lambda}}(\tilde{\boldsymbol{\Phi}} \mid \tilde{\boldsymbol{\Lambda}}) d \tilde{\boldsymbol{\Phi}} \\
& =\int p_{\boldsymbol{\Theta}^{\prime}}(\tilde{\boldsymbol{\Theta}}) p_{\boldsymbol{\Phi} \mid \boldsymbol{\Lambda}}(\tilde{\boldsymbol{\Phi}} \mid \tilde{\mathbf{\Lambda}}) d \tilde{\boldsymbol{\Phi}} \\
& =p_{\boldsymbol{\Theta}^{\prime}}(\tilde{\boldsymbol{\Theta}}) \\
& =p_{\boldsymbol{\Theta}}(\tilde{\boldsymbol{\Theta}}) \text {, }
\end{aligned}
$$

where (78) and (79) follow by the same reasoning as (73) and (74), respectively, and (81) comes from the fact that $\boldsymbol{\Theta}$ and $\boldsymbol{\Theta}^{\prime}$ have the same distribution. By (75) and (81), we conclude that $\Theta$ is an i.d. unitary matrix and is independent of $\boldsymbol{\Lambda}$, completing the proof of Lemma 5. 


\section{APPENDIX B}

PROOF OF LEMMA 6

To show (31), we first define a function $\Pi: S \rightarrow S$ with $S=$ $\{1, \ldots, m\}$ as follows to order the row vectors of $\mathbf{M}$

$$
\begin{aligned}
& \Pi(1)=\arg \max _{i \in S} h\left(\mathbf{M}_{i}\right), \\
& \Pi(2)=\arg \max _{i \in S \backslash\{\Pi(1)\}} h\left(\mathbf{M}_{i} \mid \mathbf{M}_{\Pi(1)}\right), \\
& \Pi(3)=\arg \max _{i \in S \backslash\{\Pi(1), \Pi(2)\}} h\left(\mathbf{M}_{i} \mid \mathbf{M}_{\Pi(1)}, \mathbf{M}_{\Pi(2)}\right), \\
& \vdots \\
& \Pi(m-1)=\arg \max _{i \in S \backslash\left\{\bigcup_{j=1}^{m-2} \Pi(j)\right\}} h\left(\mathbf{M}_{i} \mid \bigcup_{j=1}^{m-2} \mathbf{M}_{\Pi(j)}\right), \\
& \Pi(m)=\arg \max _{i \in S \backslash\left\{\bigcup_{j=1}^{m-1} \Pi(j)\right\}} h\left(\mathbf{M}_{i} \mid \bigcup_{j=1}^{m-1} \mathbf{M}_{\Pi(j)}\right),
\end{aligned}
$$

where $\mathbf{M}_{i}$ denotes the $i$ th row vector of $\mathbf{M}$. Note that if we order the row vectors by this function $\Pi$, we have

$$
\begin{aligned}
h\left(\mathbf{M}_{\Pi(k)} \mid \bigcup_{j=1}^{k-1} \mathbf{M}_{\Pi(j)}\right) & \geq h\left(\mathbf{M}_{\Pi(k+1)} \mid \bigcup_{j=1}^{k-1} \mathbf{M}_{\Pi(j)}\right) \\
& \geq h\left(\mathbf{M}_{\Pi(k+1)} \mid \bigcup_{j=1}^{k} \mathbf{M}_{\Pi(j)}\right),
\end{aligned}
$$

for all $k \in\{1, \ldots, m-1\}$, where (82) comes from the definition of $\Pi$, and (83) is due to the fact that the additional conditioning on $\mathbf{M}_{\Pi(k)}$ will reduce the differential entropy. The inequality in (83) implies that the conditional differential entropy $h\left(\mathbf{M}_{\Pi(k)} \mid \bigcup_{j=1}^{k-1} \mathbf{M}_{\Pi(j)}\right)$ is non-increasing with respect to the index $k$.

Now, for any given number $n<m$, we can select $\mathrm{p}_{1}=$ $\left\{\bigcup_{j=1}^{n} \Pi(j)\right\}$ and $\mathrm{p}_{2}=\left\{\bigcup_{j=n+1}^{m} \Pi(j)\right\}$ which form a partition of $S=\{1, \ldots, m\}$. We have

$$
\begin{aligned}
h\left(\mathbf{M}_{\mathrm{p}_{2}} \mid \mathbf{M}_{\mathrm{p}_{1}}\right) & =\sum_{k=n+1}^{m} h\left(\mathbf{M}_{\Pi(k)} \mid \bigcup_{j=1}^{k-1} \mathbf{M}_{\Pi(j)}\right) \\
& \leq(m-n) h\left(\mathbf{M}_{\Pi(n+1)} \mid \bigcup_{j=1}^{n} \mathbf{M}_{\Pi(j)}\right) \\
& \leq(m-n) \frac{1}{n} \sum_{k=1}^{n} h\left(\mathbf{M}_{\Pi(k)} \mid \bigcup_{j=1}^{k-1} \mathbf{M}_{\Pi(j)}\right) \\
& =\frac{(m-n)}{n} h\left(\mathbf{M}_{\mathrm{p}_{1}}\right),
\end{aligned}
$$

where (84) comes from the chain rule of differential entropy; and both (85) and (86) follow from (83). More specifically, (85) follows from the fact that the largest term inside the summation of (84) is the conditional differential entropy with index $k=n+$ 1 and (86) follows from the fact that the conditional differential entropy with index $k=n+1$ is smaller than each term inside the summation of (86). Finally, by adding $(m-n) h\left(\mathbf{M}_{\mathrm{p}_{2}} \mid \mathbf{M}_{\mathrm{p}_{1}}\right) / n$ to both sides of (87), we obtain

$$
\frac{m}{n} h\left(\mathbf{M}_{\mathrm{p}_{2}} \mid \mathbf{M}_{\mathrm{p}_{1}}\right) \leq \frac{m-n}{n}\left(h\left(\mathbf{M}_{\mathrm{p}_{1}}\right)+h\left(\mathbf{M}_{\mathrm{p}_{2}} \mid \mathbf{M}_{\mathrm{p}_{1}}\right)\right)
$$

which results in (31), completing the proof of Lemma 6.

\section{APPENDIX C \\ PROOF OF LEMMA 7}

Before showing the proof of Lemma 7, we first introduce some background from [22]. A $n \times T$ matrix $\mathbf{M}$ where $T \geq n$, can be represented by a change of coordinate system as

$$
\mathbf{M} \rightarrow\left(\mathbf{\Omega}_{\mathbf{M}}, \mathbf{C}_{\mathbf{M}}\right),
$$

where the subspace $\boldsymbol{\Omega}_{\mathbf{M}}$ is generated by its own row vectors $\mathbf{M}_{i} s, \forall i \in\{1, \ldots, n\}$, and the $n \times n$ matrix $\mathbf{C}_{\mathbf{M}}$ represents each row vector $\mathbf{M}_{i}$ with respect to an orthonormal basis of $\boldsymbol{\Omega}_{\mathbf{M}}$. The mapping in (89) changes the coordinate system of matrix $\mathbf{M}$ from $\mathrm{C}^{n \times T}$ to $G(T, n) \times \mathrm{C}^{n \times n}$ where $G(T, n)$ is a Grassmann manifold with $n(T-n)$ d.o.f. Now we can state a result given in [22] as follows.

Lemma 15: For a random matrix $\mathbf{M} \in \mathrm{e}^{n \times T}, T \geq n$, which is i.d., the differential entropy of $\mathbf{M}$ can be written as

$$
h(\mathbf{M})=h\left(\mathbf{C}_{\mathbf{M}}\right)+\log |G(T, n)|+(T-n) \mathbb{E}\left[\log \operatorname{det} \mathbf{M} \mathbf{M}^{\dagger}\right],
$$

where the $n \times n$ matrix $\mathbf{C}_{\mathbf{M}}$ and the Grassmann manifold $G(T, n)$ are defined following (89).

To prove Lemma 7, note that due to Lemma 5, the received signal $\mathbf{Y}$ is i.d. Then, from Lemma 15, we have

$$
\begin{aligned}
h(\mathbf{Y})= & h\left(\mathbf{C}_{\mathbf{Y}}\right)+\log \left|G\left(T, n_{r}\right)\right|+\left(T-n_{r}\right) \mathbb{E}\left[\log \operatorname{det} \mathbf{Y} \mathbf{Y}^{\dagger}\right] \\
\leq & n_{r}^{2} \log \pi e\left(\frac{\left(P+\sigma_{r}^{2}\right) T}{n_{r}}\right)+\left|G\left(T, n_{r}\right)\right| \\
& +\left(T-n_{r}\right) \mathbb{E}\left[\log \operatorname{det} \mathbf{Y} \mathbf{Y}^{\dagger}\right]
\end{aligned}
$$

where the inequality comes from upper bounding $h\left(\mathbf{C}_{\mathbf{Y}}\right)$ by assuming that each element of $\mathbf{C}_{\mathbf{Y}}$ is i.i.d. Gaussian with variance $\left(P+\sigma_{r}^{2}\right) T / n_{r}$. Now, note that the volume of Grassmann manifold $G\left(T, n_{r}\right)$ is a finite constant which is independent of $P$ as

$$
|G(T, n)|=\frac{\prod_{i=T-n+1}^{T} \frac{2 \pi^{2}}{(i-1) !}}{\prod_{i=1}^{n} \frac{2 \pi^{2}}{(i-1) !}} .
$$

Combining (90) and (91) completes the proof of Lemma 7. 


\section{APPENDIX D}

PROOF OF LEMMA 8

First, we introduce a few useful lemmas from [25].

Lemma 16: If $\mathbf{M}_{a} \in \mathrm{e}^{m \times m}$ and $\mathbf{M}_{b} \in \mathrm{e}^{m \times m}$ are both Hermitian matrices, then

$$
\sum_{i=1}^{m}\left(\lambda_{i}\left(\mathbf{M}_{b}\right)-\lambda_{i}\left(\mathbf{M}_{a}\right)\right)^{2} \leq\left\|\mathbf{M}_{b}-\mathbf{M}_{a}\right\|_{2}^{2}
$$

where $\lambda_{i}(A)$ denotes the $i$ th largest eigenvalue of matrix $A$ and $\|\cdot\|_{2}$ is the Frobenius norm.

Lemma 17: Let $\mathbf{M}_{a}$ and $\mathbf{M}_{b}$ be both $m \times m$ Hermitian matrices. Assume that $\mathbf{M}_{b}-\mathbf{M}_{a}$ is positive semi-definite. We have

$$
\lambda_{i}\left(\mathbf{M}_{b}\right) \geq \lambda_{i}\left(\mathbf{M}_{a}\right), \quad \forall i \in\{1, \ldots, m\} .
$$

Lemma 18: For any matrix $\mathbf{M}_{a} \in \mathrm{e}^{m \times n}$ and $\mathbf{M}_{b} \in \mathfrak{C}^{n \times m}$ where $m \geq n$,

$$
\lambda_{i}\left(\mathbf{M}_{a} \mathbf{M}_{b}\right)=\lambda_{i}\left(\mathbf{M}_{b} \mathbf{M}_{a}\right), \quad \forall i \in\{1, \ldots, n\} .
$$

From Lemmas 16 and 17, we can infer the following result.

Corollary 1: Let $\mathbf{M}_{a}$ and $\mathbf{M}_{b}$ be both $m \times m$ Hermitian matrices and assume $\mathbf{M}_{b}-\mathbf{M}_{a}$ is positive semi-definite. Then,

$$
\lambda_{i}\left(\mathbf{M}_{b}\right)-\lambda_{i}\left(\mathbf{M}_{a}\right) \leq\left\|\mathbf{M}_{b}-\mathbf{M}_{a}\right\|_{2}, \quad \forall i \in\{1, \ldots, m\} .
$$

To derive the upper bound on $\mathbb{E}\left[\log \operatorname{det} \mathbf{Y} \mathbf{Y}^{\dagger}\right]$ given in Lemma 8, we first recall that, given $\mathbf{X}=\tilde{\mathbf{X}}$, where $\tilde{\mathbf{X}}$ denotes a realization of $\mathbf{X}$, the row vectors of $\mathbf{Y}$ are i.i.d. Gaussian vectors with covariance matrix $\tilde{\mathbf{X}}^{\dagger} \tilde{\mathbf{X}}+\sigma_{r}^{2} \mathbf{I}_{T}$. Let $\mathbf{Q}_{n_{r}, T} \in \mathfrak{C}^{n_{r} \times T}$ be a random matrix whose elements are i.i.d. complex Gaussian variables with zero-mean and unit-variance. Then, we have

$$
(\mathbf{Y} \mid \mathbf{X}=\tilde{\mathbf{X}}) \stackrel{d}{\sim} \mathbf{Q}_{n_{r}, T}\left(\tilde{\mathbf{X}}^{\dagger} \tilde{\mathbf{X}}+\sigma_{r}^{2} \mathbf{I}_{T}\right)^{1 / 2}
$$

where $\mathbf{A} \stackrel{d}{\sim} \mathbf{B}$ denotes $\mathbf{A}$ has the same distribution as $\mathbf{B}$. Therefore, we have

$$
\left(\mathbf{Y} \mathbf{Y}^{\dagger} \mid \mathbf{X}=\tilde{\mathbf{X}}\right) \stackrel{d}{\sim} \mathbf{Q}_{n_{r}, T}\left(\tilde{\mathbf{X}}^{\dagger} \tilde{\mathbf{X}}+\sigma_{r}^{2} \mathbf{I}_{T}\right) \mathbf{Q}_{n_{r}, T}^{\dagger} .
$$

From Lemma 5, we know that the optimal input can be written as $\mathbf{X}=\Lambda \Theta$ where $\Lambda \in \mathrm{e}^{n_{t} \times T}$ is a diagonal random matrix and $\boldsymbol{\Theta} \in \mathcal{C}^{T \times T}$ is an i.d. unitary matrix. Hence, by taking $\tilde{\mathbf{X}}=\tilde{\boldsymbol{\Lambda}} \tilde{\boldsymbol{\Theta}}$, we can rewrite (97) as follows

$$
\begin{gathered}
\left(\mathbf{Y} \mathbf{Y}^{\dagger} \mid \mathbf{X}=\tilde{\mathbf{X}}\right) \\
\stackrel{d}{\sim} \mathbf{Q}_{n_{r}, T}\left(\tilde{\boldsymbol{\Theta}}^{\dagger} \tilde{\Lambda}^{\dagger} \tilde{\boldsymbol{\Lambda}} \tilde{\boldsymbol{\Theta}}+\sigma_{r}^{2} \mathbf{I}_{T}\right) \mathbf{Q}_{n_{r}, T}^{\dagger} \\
\stackrel{d}{\sim} \mathbf{Q}_{n_{r}, T} \tilde{\boldsymbol{\Theta}}^{\dagger}\left(\operatorname{diag}\left(\left\|\tilde{\mathbf{X}}_{1}\right\|^{2}, \ldots,\left\|\tilde{\mathbf{X}}_{n_{t}}\right\|^{2}, 0, \ldots, 0\right)\right. \\
\left.\quad+\sigma_{r}^{2} \mathbf{I}_{T}\right) \tilde{\boldsymbol{\Theta}} \mathbf{Q}_{n_{r}, T}^{\dagger} \\
\stackrel{d}{\sim} \mathbf{Q}_{a}\left(\tilde{\mathbf{\Lambda}}_{x}^{2}+\sigma_{r}^{2} \mathbf{I}_{n_{t}}\right) \mathbf{Q}_{a}^{\dagger}+\sigma_{r}^{2} \mathbf{Q}_{b} \mathbf{Q}_{b}^{\dagger},
\end{gathered}
$$

where $\tilde{\mathbf{\Lambda}}_{x} \triangleq \operatorname{diag}\left(\left\|\tilde{\mathbf{X}}_{1}\right\|,\left\|\tilde{\mathbf{X}}_{2}\right\|, \ldots,\left\|\tilde{\mathbf{X}}_{n_{t}}\right\|\right)$. In the above, (100) comes from the fact that the i.i.d. Gaussian random matrix $\mathbf{Q}_{n_{r}, T}$ is i.d. with $\left[\mathbf{Q}_{a} \mid \mathbf{Q}_{b}\right]=\mathbf{Q}_{n_{r}, T}$, where $\mathbf{Q}_{a} \in \mathrm{e}^{n_{r} \times n_{t}}$ contains the first $n_{t}$ columns of $\mathbf{Q}_{n_{r}, T}$ and $\mathbf{Q}_{b} \in \mathcal{C}^{n_{r} \times\left(T-n_{t}\right)}$ contains the remaining $\left(T-n_{t}\right)$ columns of $\mathbf{Q}_{n_{r}, T}$. To simplify the notation, let

$$
\begin{aligned}
& \mathbf{B}=\mathbf{Q}_{a}\left(\tilde{\boldsymbol{\Lambda}}_{x}^{2}+\sigma_{r}^{2} \mathbf{I}_{n_{t}}\right) \mathbf{Q}_{a}^{\dagger}+\sigma_{r}^{2} \mathbf{Q}_{b} \mathbf{Q}_{b}^{\dagger}, \\
& \mathbf{A}=\mathbf{Q}_{a}\left(\tilde{\boldsymbol{\Lambda}}_{x}^{2}+\sigma_{r}^{2} \mathbf{I}_{n_{t}}\right) \mathbf{Q}_{a}^{\dagger}
\end{aligned}
$$

and we have

$$
\begin{aligned}
\mathbb{E} & {\left[\log \operatorname{det} \mathbf{Y} \mathbf{Y}^{\dagger}\right] } \\
& =\mathbb{E}_{\mathbf{X}} \mathbb{E}\left[\log \operatorname{det} \mathbf{Y} \mathbf{Y}^{\dagger} \mid \mathbf{X}=\tilde{\mathbf{X}}\right] \\
& =\mathbb{E}_{\mathbf{X}} \mathbb{E}\left[\log \prod_{i=1}^{n_{r}} \lambda_{i}(\mathbf{B})\right] \\
& \leq \mathbb{E}_{\mathbf{X}} \sum_{i=1}^{n_{r}} \mathbb{E}_{\mathbf{Q}_{a}, \mathbf{Q}_{b}}\left[\log \left(\lambda_{i}(\mathbf{A})+\sigma_{r}^{2}\left\|\mathbf{Q}_{b} \mathbf{Q}_{b}^{\dagger}\right\|_{2}\right)\right],
\end{aligned}
$$

where (105) follows from Corollary 1. Then, by using Jensen's inequality on $\mathbf{Q}_{b}$, and the definition of $\mathbf{A}$ in (102), we get the following for the right-hand-side of (105),

$$
\begin{aligned}
& \mathbb{E}_{\mathbf{X}} \sum_{i=1}^{n_{r}} \mathbb{E}_{\mathbf{Q}_{a}, \mathbf{Q}_{b}}\left[\log \left(\lambda_{i}(\mathbf{A})+\sigma_{r}^{2}\left\|\mathbf{Q}_{b} \mathbf{Q}_{b}^{\dagger}\right\|_{2}\right)\right] \\
& \leq \mathbb{E}_{\mathbf{X}} \sum_{i=1}^{n_{r}} \mathbb{E}_{\mathbf{Q}_{a}}\left[\log \left(\lambda_{i}\left(\mathbf{Q}_{a}\left(\boldsymbol{\Lambda}_{x}^{2}+\sigma_{r}^{2} \mathbf{I}_{n_{t}}\right) \mathbf{Q}_{a}^{\dagger}\right)+\sigma_{r}^{2} k_{1}\right)\right] \\
& =\mathbb{E}_{\mathbf{X}} \mathbb{E}_{\mathbf{Q}_{a}}\left[\log \operatorname{det}\left(\mathbf{Q}_{a}\left(\boldsymbol{\Lambda}_{x}^{2}+\sigma_{r}^{2} \mathbf{I}_{n_{t}}\right) \mathbf{Q}_{a}^{\dagger}+\sigma_{r}^{2} k_{1} \mathbf{I}_{n_{r}}\right)\right] \\
& =\mathbb{E}_{\mathbf{Q}_{a}} \mathbb{E}_{\mathbf{X}}\left[\log \operatorname{det}\left(\mathbf{Q}_{a}\left(\boldsymbol{\Lambda}_{x}^{2}+\sigma_{r}^{2} \mathbf{I}_{n_{t}}\right) \mathbf{Q}_{a}^{\dagger}+\sigma_{r}^{2} k_{1} \mathbf{I}_{n_{r}}\right)\right] \\
& \leq \mathbb{E}_{\mathbf{Q}_{a}}\left[\log \operatorname{det}\left(\mathbf{Q}_{a}\left(\mathbb{E}_{\mathbf{X}}\left[\boldsymbol{\Lambda}_{x}^{2}\right]+\sigma_{r}^{2} \mathbf{I}_{n_{t}}\right) \mathbf{Q}_{a}^{\dagger}+\sigma_{r}^{2} k_{1} \mathbf{I}_{n_{r}}\right)\right]
\end{aligned}
$$

where $\quad \boldsymbol{\Lambda}_{x}=\operatorname{diag}\left(\left\|\mathbf{X}_{1}\right\|,\left\|\mathbf{X}_{2}\right\|, \ldots,\left\|\mathbf{X}_{n_{t}}\right\|\right), \quad$ and $\quad k_{1}=$ $\mathbb{E}\left[\left\|\mathbf{Q}_{b} \mathbf{Q}_{b}^{\dagger}\right\|_{2}\right]$ in (106) is a finite constant independent of $P$, the exchange of expectation over $\mathbf{X}$ and $\mathbf{Q}_{a}$ in (108) follows from the fact that $\mathbf{X}$ and $\mathbf{Q}_{a}$ are independent, and (109) comes from applying Jensen's inequality on $\mathbf{X}$.

Note that the right-hand-side of (109) is a concave function of $\mathbb{E}_{\mathbf{X}}\left[\mathbf{\Lambda}_{x}^{2}\right]$ and, since the distribution of $\mathbf{Q}_{a}$ is invariant to the permutation of its rows, it can be shown that the RHS of (109) is also a symmetric function with respect to the diagonal entries of $\mathbb{E}_{\mathbf{X}}\left[\boldsymbol{\Lambda}_{x}^{2}\right]$, where a symmetric function is defined as a function that is invariant to permutations of its input variables. These properties imply, from [26], that (109) is a Schur-concave function with respect to the diagonal entries of $\mathbb{E}_{\mathbf{X}}\left[\boldsymbol{\Lambda}_{x}^{2}\right]$. Recall the definition of Schur-concave functions as follows. 
Definition 1: A function $f: \mathcal{R}^{n} \rightarrow \mathcal{R}$ is said to be a Schurconcave function if, for any $\mathbf{x}$ and $\mathbf{y}$ such that $\mathbf{x} \prec \mathbf{y}$ (i.e., $\mathbf{x}$ is majorized by $\mathbf{y})$ [26], we have $f(\mathbf{x}) \geq f(\mathbf{y})$.

Note that $\operatorname{det}(\cdot)$ is matrix nondecreasing on the set of positive semi-definite matrices [27, Section 3.6.1] (i.e., $\operatorname{det}\left(\mathbf{M}_{a}\right) \geq$ $\operatorname{det}\left(\mathbf{M}_{b}\right)$, for all $\mathbf{M}_{a}, \mathbf{M}_{b}$, and $\mathbf{M}_{a}-\mathbf{M}_{b}$ that are positive semi-definite). This implies that, under the power constraint $\operatorname{tr}\left(\mathbb{E}_{\mathbf{X}}\left[\Lambda_{x}^{2}\right]\right) \leq P T$, the RHS of (109) can be upper-bounded by taking $\mathbb{E}_{\mathbf{X}}\left[\Lambda_{x}^{2}\right]$ that satisfies $\operatorname{tr}\left(\mathbb{E}_{\mathbf{X}}\left[\boldsymbol{\Lambda}_{x}^{2}\right]\right)=P T$. Moreover, since the diagonal entries of $\left(P T / n_{t}\right) \mathbf{I}_{n_{t}}$ form a vector that is majorized by all vectors summing up to $P T$, it follows by the property of Schur-concave functions that the RHS of (109) can be further upper bounded by choosing $\mathbb{E}_{\mathbf{X}}\left[\boldsymbol{\Lambda}_{x}^{2}\right]=\left(P T / n_{t}\right) \mathbf{I}_{n_{t}}$, Hence, we have

$$
\begin{aligned}
& \mathbb{E}\left[\log \operatorname{det} \mathbf{Y} \mathbf{Y}^{\dagger}\right] \\
& \leq \mathbb{E}_{\mathbf{Q}_{a}}\left[\log \operatorname{det}\left(\mathbf{Q}_{a}\left(\frac{P T}{n_{t}} \mathbf{I}_{n_{t}}+\sigma_{r}^{2} \mathbf{I}_{n_{t}}\right) \mathbf{Q}_{a}^{\dagger}+\sigma_{r}^{2} k_{1} \mathbf{I}_{n_{r}}\right)\right] \\
& =\mathbb{E}_{\mathbf{Q}_{a}}\left[\log \operatorname{det}\left(\left(\frac{P T}{n_{t}}+\sigma_{r}^{2}\right) \mathbf{Q}_{a} \mathbf{Q}_{a}^{\dagger}+\sigma_{r}^{2} k_{1} \mathbf{I}_{n_{r}}\right)\right] \\
& =\mathbb{E}_{\mathbf{Q}_{a}}\left[\log \operatorname{det}\left(\left(\frac{P T}{n_{t}}+\sigma_{r}^{2}\right) \mathbf{I}_{n_{r}}+\sigma_{r}^{2} k_{1}\left(\mathbf{Q}_{a} \mathbf{Q}_{a}^{\dagger}\right)^{-1}\right)\right]+k_{2} \\
& \leq \mathbb{E}_{\mathbf{Q}_{a}}\left[\operatorname { l o g } \operatorname { d e t } \left(\left(\frac{P T}{n_{t}}+\sigma_{r}^{2}\right) \mathbf{I}_{n_{r}}+\sigma_{r}^{2} k_{1}\right.\right. \\
& \left.\quad \leq \log \operatorname{det}\left(\left(\frac{P T}{n_{t}}+\sigma_{r}^{2} \mathbf{Q}_{a}^{\dagger}\right)^{-1} \mathbf{I}_{n_{r}}+\sigma_{r}^{2} k_{3} \mathbf{I}_{n_{r}}\right)\right]+k_{2} \\
& \quad=n_{r} \log \left(\frac{P T}{n_{t}}+\sigma_{r}^{2}\left(k_{3}+1\right)\right)+k_{2},
\end{aligned}
$$

where $k_{2}=\mathbb{E}\left[\log \operatorname{det} \mathbf{Q}_{a} \mathbf{Q}_{a}^{\dagger}\right]$ and $k_{3}=\mathbb{E}\left[k_{1}\left\|\left(\mathbf{Q}_{a} \mathbf{Q}_{a}^{\dagger}\right)^{-1}\right\|_{2}\right]$. In the above, (113) follows from Corollary 1 since $\sigma_{r}^{2} k_{1}\left(\mathbf{Q}_{a} \mathbf{Q}_{a}^{\dagger}\right)^{-1}$ is Hermitian and positive semi-definite, and (114) follows from Jensen's inequality. Note that both $k_{2}$ and $k_{3}$ are finite constants that are independent of $P$. From (115), it follows that

$$
\max _{p_{\mathbf{X}} \in S_{P_{\mathbf{X}}}^{*}} \mathbb{E}\left[\log \operatorname{det} \mathbf{Y} \mathbf{Y}^{\dagger}\right] \leq n_{r} \log P+o(\log P),
$$

which concludes the proof of Lemma 8.

Note that, in [22] where the conventional MIMO channel was examined, it was sufficient to consider only the case where $n_{t}=$ $n_{r}$ since, in the absence of eavesdroppers, increasing the number of transmit antennas does not improve the capacity in the high SNR regime. In this case, one can more easily rewrite (109) as

$$
\begin{aligned}
\mathbb{E}_{\mathbf{Q}_{a}}\left[\operatorname { l o g } \operatorname { d e t } \left(\left(\mathbb{E}_{\mathbf{X}}\left[\boldsymbol{\Lambda}_{x}^{2}\right]\right.\right.\right. & \left.\left.\left.+\sigma_{r}^{2} \mathbf{I}_{n_{t}}\right)+\sigma_{r}^{2} k_{1}\left(\mathbf{Q}_{a}^{\dagger} \mathbf{Q}_{a}\right)^{-1}\right)\right] \\
& +\mathbb{E}_{\mathbf{Q}_{a}}\left[\log \operatorname{det} \mathbf{Q}_{a}^{\dagger} \mathbf{Q}_{a}\right]+\log \left(\sigma_{r}^{2} k_{1}\right)^{n_{r}-n_{t}},
\end{aligned}
$$

due to the invertibility of $\mathbf{Q}_{a}^{\dagger} \mathbf{Q}_{a}$. The remaining derivations are similar to that below (112). In wiretap channels, the problem does not reduce to the case where $n_{t}=n_{r}$ and, thus, a new upper-bounding technique was needed to cope with the singularity of $\mathbf{Q}_{a}^{\dagger} \mathbf{Q}_{a}$ when $n_{t}>n_{r}$.

\section{APPENDIX E}

PROOF OF LEMMA 9

By (108) in Appendix D and by further replacing the determinant by the product of eigenvalues, we have

$$
\begin{aligned}
& \mathbb{E}\left[\log \operatorname{det} \mathbf{Y} \mathbf{Y}^{\dagger}\right] \\
& \leq \mathbb{E}_{\mathbf{Q}_{a}} \mathbb{E}_{\mathbf{X}}\left[\log \operatorname{det}\left(\mathbf{Q}_{a}\left(\boldsymbol{\Lambda}_{x}^{2}+\sigma_{r}^{2} \mathbf{I}_{n_{t}}\right) \mathbf{Q}_{a}^{\dagger}+\sigma_{r}^{2} k_{1} \mathbf{I}_{n_{r}}\right)\right] \\
& =\mathbb{E}_{\mathbf{Q}_{a}} \sum_{i=1}^{n_{r}} \mathbb{E}_{\mathbf{X}}\left[\log \lambda_{i}\left(\mathbf{Q}_{a}\left(\boldsymbol{\Lambda}_{x}^{2}+\sigma_{r}^{2} \mathbf{I}_{n_{t}}\right) \mathbf{Q}_{a}^{\dagger}\right)+\sigma_{r}^{2} k_{1}\right] .
\end{aligned}
$$

Let $\mathbf{D}_{x} \triangleq \Lambda_{x}^{2}+\sigma_{r}^{2} \mathbf{I}_{n_{t}}$. Note that $\mathbf{D}_{x}$ is a real diagonal matrix. From Lemma 18, we know that

$$
\lambda_{i}\left(\mathbf{Q}_{a} \mathbf{D}_{x} \mathbf{Q}_{a}^{\dagger}\right)=\lambda_{i}\left(\mathbf{D}_{x}^{1 / 2} \mathbf{Q}_{a}^{\dagger} \mathbf{Q}_{a} \mathbf{D}_{x}^{1 / 2}\right),
$$

for all $i \in\left\{1, \ldots, n_{r}\right\}$. Let $\mathbf{Q}_{n_{t}, n_{t}}=\left[\mathbf{Q}_{a}^{T} \mid \mathbf{Q}_{c}^{T}\right]^{T}$, where $\mathbf{Q}_{c} \in$ $\mathrm{C}^{\left(n_{t}-n_{r}\right) \times n_{t}}$ such that $\mathbf{Q}_{n_{t}, n_{t}}$ is a matrix with entries that are i.i.d. Gaussian with zero-mean and unit-variance. Then, we have

$$
\mathbf{D}_{x}^{1 / 2} \mathbf{Q}_{n_{t}, n_{t}}^{\dagger} \mathbf{Q}_{n_{t}, n_{t}} \mathbf{D}_{x}^{1 / 2}=\mathbf{D}_{x}^{1 / 2} \mathbf{Q}_{a}^{\dagger} \mathbf{Q}_{a} \mathbf{D}_{x}^{1 / 2}+\mathbf{D}_{x}^{1 / 2} \mathbf{Q}_{c}^{\dagger} \mathbf{Q}_{c} \mathbf{D}_{x}^{1 / 2} .
$$

By the above, we can further upper bound (118) as

$$
\begin{aligned}
& \mathbb{E}\left[\log \operatorname{det} \mathbf{Y} \mathbf{Y}^{\dagger}\right] \\
& \leq \mathbb{E}_{\mathbf{Q}_{a}} \sum_{i=1}^{n_{r}} \mathbb{E}_{\mathbf{X}}\left[\log \left(\lambda_{i}\left(\mathbf{D}_{x}^{1 / 2} \mathbf{Q}_{a}^{\dagger} \mathbf{Q}_{a} \mathbf{D}_{x}^{1 / 2}\right)+\sigma_{r}^{2} k_{1}\right)\right] \\
& \leq \mathbb{E}_{\mathbf{Q}_{n_{t}, n_{t}}} \sum_{i=1}^{n_{r}} \mathbb{E}_{\mathbf{X}}\left[\operatorname { l o g } \left(\lambda_{i}\left(\mathbf{D}_{x}^{1 / 2} \mathbf{Q}_{n_{t}, n_{t}}^{\dagger} \mathbf{Q}_{n_{t}, n_{t}} \mathbf{D}_{x}^{1 / 2}\right)\right.\right. \\
& \left.\left.+\sigma_{r}^{2} k_{1}\right)\right] \\
& \leq \mathbb{E}_{\mathbf{Q}_{n_{t}, n_{t}}} \sum_{i=1}^{n_{t}} \mathbb{E}_{\mathbf{X}}\left[\operatorname { l o g } \left(\lambda_{i}\left(\mathbf{D}_{x}^{1 / 2} \mathbf{Q}_{n_{t}, n_{t}}^{\dagger} \mathbf{Q}_{n_{t}, n_{t}} \mathbf{D}_{x}^{1 / 2}\right)\right.\right. \\
& \left.\left.+1+\sigma_{r}^{2} k_{1}\right)\right]
\end{aligned}
$$

where (122) comes from (120) and Lemma 17 since $\mathbf{D}_{x}^{1 / 2} \mathbf{Q}_{c}^{\dagger} \mathbf{Q}_{c} \mathbf{D}_{x}^{1 / 2}$ is Hermitian and positive semi-definite, and (123) follows by summing over the additional terms $n_{t}+1$ to $n_{r}$ with a 1 added inside each logarithm to ensure that $\log \left(\lambda_{i}\left(\mathbf{D}_{x}^{1 / 2} \mathbf{Q}_{n_{t}, n_{t}}^{\dagger} \mathbf{Q}_{n_{t}, n_{t}} \mathbf{D}_{x}^{1 / 2}\right)+1+\sigma_{r}^{2} k_{1}\right)>0$, for all $i \in\left\{n_{t}-\right.$ 
$\left.n_{r}+1, \ldots, n_{t}\right\}$, since $k_{1} \sigma_{r}^{2}>0$ and each eigenvalue is nonnegative. Moreover, by rewriting (123), we have

$$
\begin{aligned}
& \mathbb{E}\left[\log \operatorname{det} \mathbf{Y} \mathbf{Y}^{\dagger}\right] \\
& \leq \mathbb{E}_{\mathbf{Q}_{n_{t}, n_{t}}} \sum_{i=1}^{n_{t}} \mathbb{E}_{\mathbf{X}}\left[\log \left(\lambda_{i}\left(\mathbf{D}_{x} \mathbf{Q}_{n_{t}, n_{t}}^{\dagger} \mathbf{Q}_{n_{t}, n_{t}}\right)+1+\sigma_{r}^{2} k_{1}\right)\right] \\
& =\mathbb{E}_{\mathbf{Q}_{n_{t}, n_{t}}} \mathbb{E}_{\mathbf{X}}\left[\log \operatorname{det}\left(\mathbf{D}_{x} \mathbf{Q}_{n_{t}, n_{t}}^{\dagger} \mathbf{Q}_{n_{t}, n_{t}}+\left(1+\sigma_{r}^{2} k_{1}\right) \mathbf{I}_{n_{t}}\right)\right] \\
& =\mathbb{E}_{\mathbf{Q}_{n_{t}, n_{t}}} \mathbb{E}_{\mathbf{X}}\left[\operatorname { l o g } \operatorname { d e t } \left(\mathbf{D}_{x}+\left(1+\sigma_{r}^{2} k_{1}\right)\right.\right. \\
& \left.\left.\cdot\left(\mathbf{Q}_{n_{t}, n_{t}}^{\dagger} \mathbf{Q}_{n_{t}, n_{t}}\right)^{-1}\right)\right]+k_{4} \\
& \leq \mathbb{E}_{\mathbf{X}}\left[\operatorname { l o g } \operatorname { d e t } \left(\mathbf{D}_{x}+\left(1+\sigma_{r}^{2} k_{1}\right)\right.\right. \\
& \left.\left.\cdot \mathbb{E}_{\mathbf{Q}_{n_{t}, n_{t}}}\left[\left\|\left(\mathbf{Q}_{n_{t}, n_{t}}^{\dagger} \mathbf{Q}_{n_{t}, n_{t}}\right)^{-1}\right\|_{2}\right] \mathbf{I}_{n_{t}}\right)\right]+k_{4} \\
& \leq \mathbb{E}_{\mathbf{X}}\left[\log \operatorname{det}\left(\boldsymbol{\Lambda}_{x}^{2}+k_{5} \mathbf{I}_{n_{t}}\right)\right]+k_{4} \\
& =\sum_{i=1}^{n_{t}} \mathbb{E}\left[\log \left(\left\|\mathbf{X}_{i}\right\|^{2}+k_{5}\right)\right]+k_{4}
\end{aligned}
$$

where $k_{4}=\mathbb{E}\left[\log \operatorname{det} \mathbf{Q}_{n_{t}, n_{t}}^{\dagger} \mathbf{Q}_{n_{t}, n_{t}}\right] \quad$ and $\quad k_{5}=\sigma_{r}^{2}+(1+$ $\left.\sigma_{r}^{2} k_{1}\right) \mathbb{E}\left[\left\|\left(\mathbf{Q}_{n_{t}, n_{t}}^{\dagger} \mathbf{Q}_{n_{t}, n_{t}}\right)^{-1}\right\|_{2}\right]$, and the derivation of (127) from (126) follows similarly to that of (105) from (104). Finally, from the upper bound of $\mathbb{E}\left[\log \operatorname{det} \mathbf{Y} \mathbf{Y}^{\dagger}\right]$ in (129), we have

$$
\begin{aligned}
& \mathbb{E}\left[\log \operatorname{det} \mathbf{Y} \mathbf{Y}^{\dagger}\right]-\sum_{i=1}^{n_{t}} \mathbb{E}\left[\log \left(\left\|\mathbf{X}_{i}\right\|^{2}+\sigma_{r}^{2}\right)\right] \\
& \leq \sum_{i=1}^{n_{t}} \mathbb{E}\left[\log \left(\left\|\mathbf{X}_{i}\right\|^{2}+k_{5}\right)\right]+k_{4}-\sum_{i=1}^{n_{t}} \mathbb{E}\left[\log \left(\left\|\mathbf{X}_{i}\right\|^{2}+\sigma_{r}^{2}\right)\right] \\
& =\sum_{i=1}^{n_{t}} \mathbb{E}\left[\log \left(\frac{\left\|\mathbf{X}_{i}\right\|^{2}+k_{5}}{\left\|\mathbf{X}_{i}\right\|^{2}+\sigma_{r}^{2}}\right)\right]+k_{4} \\
& \leq \sum_{i=1}^{n_{t}} \mathbb{E}\left[\left(\frac{k_{5}-\sigma_{r}^{2}}{\left\|\mathbf{X}_{i}\right\|^{2}+\sigma_{r}^{2}}\right)\right]+k_{4} \\
& \leq n_{t}\left(\frac{k_{5}-\sigma_{r}^{2}}{\sigma_{r}^{2}}\right)+k_{4}
\end{aligned}
$$

where (132) comes from $k_{5}>\sigma_{r}^{2}$ by definition and $\log (1+$ $x)<x$ when $x \geq 0$. Since $k_{4}$ in (126) and $k_{5}$ in (128) are finite constants independent of $P$, this completes the proof of Lemma 9.

Note here that, similar to Lemma 8, one cannot directly apply the results in [22] to prove Lemma 9. This is because the results in [22] rely on the invertibility of $\mathbf{Q}_{a}^{\dagger} \mathbf{Q}_{a}$, as mentioned below (116). However, this property may not hold here and, thus, new techniques were needed to upper bound $\mathbb{E}\left[\log \operatorname{det} \mathbf{Y} \mathbf{Y}^{\dagger}\right]$ as presented above.

\section{APPENDIX F}

\section{PROOF OF LEMMA 10}

Let $\mathbf{Y}_{n_{t}}, \mathbf{H}_{n_{t}}$, and $\mathbf{N}_{r, n_{t}}$ be matrices formed by taking $n_{t}$ rows arbitrarily from $\mathbf{Y}, \mathbf{H}$, and $\mathbf{N}_{r}$, respectively, and let $\mathbf{Y}_{n_{r}-n_{t}}$, $\mathbf{H}_{n_{r}-n_{t}}$, and $\mathbf{N}_{r, n_{r}-n_{t}}$ be the remaining $\left(n_{r}-n_{t}\right)$ rows of $\mathbf{Y}, \mathbf{H}$, and $\mathbf{N}_{r}$, respectively. Thus, we have

$$
\begin{aligned}
\mathbf{Y}_{n_{t}} & =\mathbf{H}_{n_{t}} \mathbf{X}+\mathbf{N}_{r, n_{t}}, \\
\mathbf{Y}_{n_{r}-n_{t}} & =\mathbf{H}_{n_{r}-n_{t}} \mathbf{X}+\mathbf{N}_{r, n_{r}-n_{t}} .
\end{aligned}
$$

First, note that, if $n_{t}<n_{r}$, we can represent the channel matrix $\mathbf{H}_{n_{r}-n_{t}}$ in terms of linear combinations of row vectors of $\mathbf{H}_{n_{t}}$. Thus, we have

$$
\mathbf{Y}_{n_{r}-n_{t}}=C(\mathbf{H}) \mathbf{Y}_{n_{t}}+\mathbf{N}_{r, n_{r}-n_{t}}-C(\mathbf{H}) \mathbf{N}_{r, n_{t}},
$$

where $C(\mathbf{H})$ is the linear combination matrix such that $C(\mathbf{H}) \mathbf{H}_{n_{t}}=\mathbf{H}_{n_{r}-n_{t}}$. From (136), the following Markov relation holds

$$
\begin{aligned}
\mathbf{X} & \rightarrow\left(\mathbf{Y}_{n_{t}}, C(\mathbf{H}), \mathbf{N}_{r, n_{r}-n_{t}}-C(\mathbf{H}) \mathbf{N}_{r, n_{t}}\right) \\
& \rightarrow\left(\mathbf{Y}_{n_{t}}, \mathbf{Y}_{n_{r}-n_{t}}\right) \rightarrow \mathbf{Y} .
\end{aligned}
$$

Hence, from the data processing inequality, we have

$$
\begin{aligned}
& I(\mathbf{X} ; \mathbf{Y})-I\left(\mathbf{X} ; \mathbf{Y}_{n_{t}}\right) \\
& \leq I\left(\mathbf{X} ; \mathbf{Y}_{n_{t}}, C(\mathbf{H}), \mathbf{N}_{r, n_{r}-n_{t}}-C(\mathbf{H}) \mathbf{N}_{r, n_{t}}\right)-I\left(\mathbf{X} ; \mathbf{Y}_{n_{t}}\right) \\
&=I\left(\mathbf{X} ; C(\mathbf{H}), \mathbf{N}_{r, n_{r}-n_{t}}-C(\mathbf{H}) \mathbf{N}_{r, n_{t}} \mid \mathbf{Y}_{n_{t}}\right) \\
&= h\left(C(\mathbf{H}), \mathbf{N}_{r, n_{r}-n_{t}}-C(\mathbf{H}) \mathbf{N}_{r, n_{t}} \mid \mathbf{Y}_{n_{t}}\right) \\
&-h\left(C(\mathbf{H}), \mathbf{N}_{r, n_{r}-n_{t}}-C(\mathbf{H}) \mathbf{N}_{r, n_{t}} \mid \mathbf{Y}_{n_{t}}, \mathbf{X}\right) .
\end{aligned}
$$

The first term in (140) can be upper bounded by

$$
\begin{aligned}
h(C(\mathbf{H}), & \left.\mathbf{N}_{r, n_{r}-n_{t}}-C(\mathbf{H}) \mathbf{N}_{r, n_{t}} \mid \mathbf{Y}_{n_{t}}\right) \\
& \leq h\left(C(\mathbf{H}), \mathbf{N}_{r, n_{r}-n_{t}}-C(\mathbf{H}) \mathbf{N}_{r, n_{t}}\right)=o(\log P),
\end{aligned}
$$

and the second term in (140) is lower bounded by

$$
\begin{aligned}
& h\left(C(\mathbf{H}), \mathbf{N}_{r, n_{r}-n_{t}}-C(\mathbf{H}) \mathbf{N}_{r, n_{t}} \mid \mathbf{Y}_{n_{t}}, \mathbf{X}\right) \\
& \geq h\left(C(\mathbf{H}), \mathbf{N}_{r, n_{r}-n_{t}}-C(\mathbf{H}) \mathbf{N}_{r, n_{t}} \mid \mathbf{Y}_{n_{t}}, \mathbf{X}, \mathbf{H}_{n_{t}}\right) \\
&= h\left(C(\mathbf{H}), \mathbf{N}_{r, n_{r}-n_{t}}-C(\mathbf{H}) \mathbf{N}_{r, n_{t}} \mid \mathbf{H}_{n_{t}}, \mathbf{X}, \mathbf{N}_{r, n_{t}}\right) \\
&= h\left(C(\mathbf{H}), \mathbf{N}_{r, n_{r}-n_{t}}-C(\mathbf{H}) \mathbf{N}_{r, n_{t}} \mid \mathbf{H}_{n_{t}}, \mathbf{N}_{r, n_{t}}\right) \\
&= h\left(C(\mathbf{H}) \mid \mathbf{H}_{n_{t}}, \mathbf{N}_{r, n_{t}}\right) \\
&+h\left(\mathbf{N}_{r, n_{r}-n_{t}}-C(\mathbf{H}) \mathbf{N}_{r, n_{t}} \mid \mathbf{H}_{n_{t}}, \mathbf{N}_{r, n_{t}}, C(\mathbf{H})\right) \\
&= h\left(C(\mathbf{H}) \mid \mathbf{H}_{n_{t}}, \mathbf{N}_{r, n_{t}}\right)+h\left(\mathbf{N}_{r, n_{r}-n_{t}}\right)=o(\log P) .
\end{aligned}
$$


The result in (56) of Lemma 10 then follows from (140), (141), and (146).

\section{REFERENCES}

[1] C. E. Shannon, "Communication theory of secrecy systems," Bell Syst. Tech. J., vol. 28, no. 4, pp. 656-715, Oct. 1949.

[2] A. D. Wyner, "The wire-tap channel," Bell Sys. Techn. J., vol. 54, no. 8, pp. 1355-1387, Oct. 1975.

[3] I. Csiszár and J. Körner, "Broadcast channels with confidential messages," IEEE Trans. Inf. Theory, vol. 24, no. 3, pp. 339-348, May 1978.

[4] S. Leung-Yan-Cheong and M. Hellman, "The Gaussian wiretap channel," IEEE Trans. Inf. Theory, vol. 24, no. 4, pp. 451-456, Jul. 1978.

[5] S. Shafiee, N. Liu, and S. Ulukus, "Towards the secrecy capacity of the Gaussian MIMO wire-tap channel: The 2-2-1 channel," IEEE Trans. Inf. Theory, vol. 55, no. 9, pp. 4033-4039, Sep. 2009.

[6] A. Khisti and G. Wornell, "Secure transmission with multiple antennas II: The MIMOME wiretap channel," IEEE Trans. Inf. Theory, vol. 56, no. 11, pp. 5515-5532, Nov. 2010.

[7] F. Oggier and B. Hassibi, "The secrecy capacity of the MIMO wiretap channel," IEEE Trans. Inf. Theory, vol. 57, no. 8, pp. 4961-4972, Aug. 2011.

[8] T. Liu and S. Shamai, "A note on the secrecy capacity of the multi-antenna wiretap channel," IEEE Trans. Inf. Theory, vol. 55, no. 6, pp. 2547-2553, Jun. 2009

[9] Y. Liang and H. V. Poor, "Secure communication over fading channels," in Proc. 44th Annu. Allerton Conf. Commun., Control, Comput., Sep. 2006, pp. 817-823.

[10] Z. Li, R. D. Yates, and W. Trappe, "Secrecy capacity of independent parallel channels," in Proc. 44th Annual Allerton Conf. Commun., Control, Comput., Sep. 2006, pp. 841-848.

[11] Y. Liang, H. V. Poor, and S. Shamai, "Secure communication over fading channels," IEEE Trans. Inf. Theory, vol. 54, no. 6, pp. 2470-2492, Jun. 2008

[12] Z. Li, R. D. Yates, and W. Trappe, "Secrecy capacity of independent parallel channels," in Securing Wireless Communications at the Physical Layer, R. Liu and W. Trappe, Eds. New York, NY, USA: SpringerVerlag, 2010, pp. 1-18.

[13] P. K. Gopala, L. Lai, and H. El Gamal, "On the secrecy capacity of fading channels," IEEE Trans. Inf. Theory, vol. 54, no. 10, pp. 4687-4698, Oct. 2008.

[14] Z. Li, R. D. Yates, and W. Trappe, "Achieving secret communication for fast rayleigh fading channels," IEEE Trans. Wireless Commun., vol. 9, no. 9, pp. 2792-2799, Sep. 2010.

[15] S.-C. Lin and C.-L. Lin, "On secrecy capacity of fast fading MIMOME wiretap channels with statistical CSIT," IEEE Trans. Wireless Commun., vol. 13, no. 6, pp. 3293-3306, Jun. 2014.

[16] X. He and A. Yener, "MIMO wiretap channels with unknown and varying eavesdropper channel states," IEEE Trans. Inf. Theory, vol. 60, no. 11, pp. 6844-6869, Nov. 2014.

[17] S. Yang, M. Kobayashi, P. Piantanida, and S. Shamai, "Secrecy degrees of freedom of MIMO broadcast channels with delayed CSIT," IEEE Trans. Inf. Theory, vol. 59, no. 9, pp. 5244-5256, Sep. 2013.

[18] L. Czap, V. M. Prabhakaran, S. Diggavi, and C. Fragouli, "Broadcasting private messages securely," in Proc. IEEE Int. Symp. Inf. Theory, Jul. 2012, pp. 428-432.

[19] G. Caire, N. Jindal, M. Kobayashi, and N. Ravindran, "Quantized vs. analog feedback for the MIMO broadcast channel: A comparison between zero forcing based achievable rates," in Proc. IEEE Int. Symp. Inf. Theory, Jun. 2007, pp. 2046-2050.

[20] P. Mukherjee and S. Ulukus, "Fading wiretap channel with no CSI anywhere," in Proc. IEEE Int. Symp. Inf. Theory, Jul. 2013, pp. 1347-1351.

[21] T. L. Marzetta and B. M. Hochwald, "Capacity of a mobile multipleantenna communication link in Rayleigh flat fading," IEEE Trans. Inf. Theory, vol. 45, no. 1, pp. 139-157, Jan. 1999.

[22] L. Zheng and D. N. C. Tse, "Communication on the Grassmann manifold: A geometric approach to the noncoherent multiple-antenna channel," IEEE Trans. Inf. Theory, vol. 48, no. 2, pp. 359-383, Feb. 2002.

[23] T. M. Cover and J. A. Thomas, Elements of Information Theory, 2nd ed. Hoboken, NJ, USA: Wiley, 2006.

[24] M. van Dijk, "On a special class of broadcast channels with confidential messages," IEEE Trans. Inf. Theory, vol. 43, no. 2, pp. 712-714, Mar. 1997.

[25] R. A. Horn and C. R. Johnson, Matrix Analysis. Cambridge, U.K.: Cambridge Univ. Press, 1990.
[26] A. W. Marshall, I. Olkin, and B. C. Arnold, Inequalities: Theory of Majorization and Its Applications, 2nd ed. New York, NY, USA: SpringerVerlag, 2011, ser. Springer series in statistics.

[27] S. Boyd and L. Vandenberghe, Convex Optimization. Cambridge, U.K. Cambridge Univ. Press, 2004.

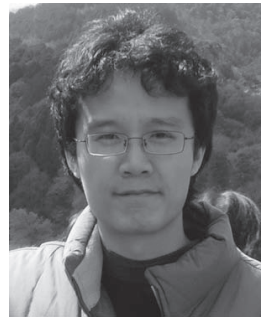

Ta-Yuan Liu received the B.S. degree in electrical engineering from National Tsing Hua University, Hsinchu, Taiwan in 2009, and is currently pursuing the Ph.D. degree in the Institute of Communications Engineering at National Tsing Hua University, Hsinchu. He was a Visiting Student at the University of Maryland, College Park, MD, USA during September 2-12 to December 2012 and July 2013 to September 2013. His research interests include physical layer security, information theory, and wireless communications.

He received the Best Student Paper Award from the National Symposium on Telecommunication (NST) in 2011.

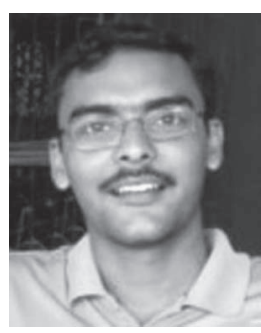

Pritam Mukherjee received the B.Tech (Hons.) degree major in electronics and electrical communication engineering and minor in computer science and engineering from Indian Institute of Technology (IIT), Kharagpur, India, in 2010. Currently, he is pursuing the Ph.D. degree in electrical and computer engineering at the University of Maryland, College Park, MD, USA, under the guidance of Prof. Sennur Ulukus. His research interests include information theoretic physical layer security and network information theory for wireless networks.

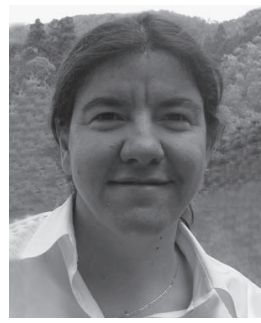

Sennur Ulukus received B.S. and M.S. degrees in electrical and electronics engineering from Bilkent University, Ankara, Turkey, and the Ph.D. degree in electrical and computer engineering from Wireless Information Network Laboratory (WINLAB), Rutgers University, New Brunswick, NJ, USA. She is a Professor of Electrical and Computer Engineering at the University of Maryland (UMD), College Park, MD, USA, where she also holds a joint appointment with the Institute for Systems Research (ISR). Prior to joining UMD, she was a Senior Technical Staff Member at AT\&T Labs-Research. Her research interests are in wireless communication theory and networking, network information theory for wireless communications, signal processing for wireless communications, information theoretic physical layer security, and energy harvesting communications.

She received the 2003 IEEE Marconi Prize Paper Award in Wireless Communications, a 2005 NSF CAREER Award, the 2010-2011 ISR Outstanding Systems Engineering Faculty Award, and the 2012 George Corcoran Education Award. She served as an Associate Editor for the IEEE TRANSACTIONS ON INFORMATION THEORY (2007-2010) and IEEE TRANSACTIONS ON COMMUNiCATIONS (2003-2007). She served as a Guest Editor for the IEEE JouRnal on SELECTED AREAS IN COMMUNiCATIONS special issue on wireless communications powered by energy harvesting and wireless energy transfer (2015), the Journal of Communications and Networks special issue on energy harvesting in wireless networks (2012), IEEE TRANSACTIONS ON INFORMATION THEORY special issue on interference networks (2011), and IEEE Journal on SELECTEd AREAS IN COMMUNICATIONS special issue on multiuser detection for advanced communication systems and networks (2008). She served as the TPC co-chair of the 2014 IEEE PIMRC, the Communication Theory Symposium at 2014 IEEE Globecom, the Communication Theory Symposium at 2013 IEEE ICC, the Physical-Layer Security Workshop at 2011 IEEE Globecom, the Physical-Layer Security Workshop at 2011 IEEE ICC, the 2011 Communication Theory Workshop (IEEE CTW), the Wireless Communications Symposium at 2010 IEEE ICC, the Medium Access Control Track at 2008 IEEE WCNC, and the Communication Theory Symposium at 2007 IEEE Globecom. She was the Secretary of the IEEE Communication Theory Technical Committee (CTTC) in 2007-2009. 


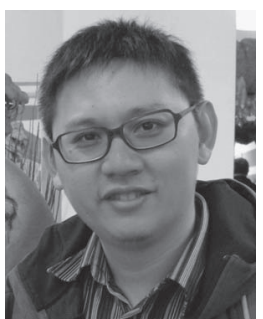

Shih-Chun Lin (M'08) received the B.S. and Ph.D. degrees in electrical engineering from National Taiwan University, Taipei, Taiwan, in 2000 and 2007, respectively. He was a Visiting Student at The Ohio State University, Columbus, OH, USA, in 2007. After finishing his military duty during 2008, he was a Postdoctoral Researcher at National Tsing Hua University, Hsinchu, Taiwan. From August 2011 to August 2012, he was an Assistant Professor at National Taipei University of Technology.

Dr. Lin is now an Assistant Professor at National Taiwan University of Science and Technology, Taipei, Taiwan. His research interests include coding/information theory, communications, and signal processing. He also served as a track chair of the International Conference on Intelligent Green Building and Smart Grid (IGBSG) 2014, and a TPC member of IEEE ICC, ICC Workshop on Wireless Physical Layer Security, and VTCSpring.

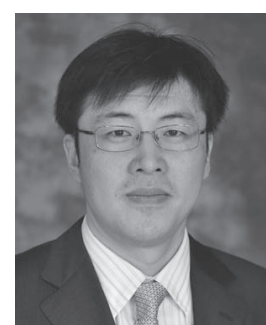

Y.-W. Peter Hong (S'01-M'05-SM'13) received the B.S. degree from National Taiwan University, Taipei, Taiwan, in 1999, and the Ph.D. degree from Cornell University, Ithaca, NY, USA, in 2005, both in electrical engineering. He joined the Institute of Communications Engineering and the Department of Electrical Engineering at National Tsing Hua University, Hsinchu, Taiwan, in Fall 2005, where he is now a Full Professor. He was also a visiting scholar at the University of Southern California during June to August 2008. His research interests include physical layer secrecy, cooperative communications, distributed signal processing for sensor networks, and cross-layer designs for wireless networks.

$\mathrm{He}$ is currently an Associate Editor for IEEE TRANSACTIONS ON SIGNAL PROCESSING and IEEE TRANSACTIONS ON INFORMATION FORENSICS AND SECURITY. He is the leading coauthor of the books Cooperative Communications and Networking: Technologies and System Design (with W.-J. Huang and C.-C. Jay Kuo) (Springer, 2010) and Signal Processing Approaches to Secure Physical Layer Communications in Multi-Antenna Wireless Systems (with P.-C. Lan and C.-C. Jay Kuo)(Springer, 2013). He served as Organizing CoChair of the 2012 IEEE Signal Processing Society Summer School on Compressive Sensing and MIMO Signal Processing Systems, Publication Co-Chair and TPC Track Co-Chair of VTC2010-Spring for the track on "Cognitive Radio and Cooperative Communications," Publicity Co-Chair of ISITA/ISSSTA 2010, and TPC Track Co-Chair of WPMC2012 for the track on Wireless Communications. He received the Best Paper Award for young authors from the IEEE IT/COM Society Taipei/Tainan Chapter in 2005, the Best Paper Award in MILCOM 2005, the Junior Faculty Research Award from the College of EECS and from National Tsing Hua University in 2009 and 2010, respectively. $\mathrm{He}$ also received the IEEE Communication Society Asia-Pacific Outstanding Young Researcher Award in 2010, the Y. Z. Hsu Scientific Paper Award, the National Science Council (NSC) Wu Ta-You Memorial Award in 2011, and the Chinese Institute of Electrical Engineering (CIEE) Outstanding Young Electrical Engineer Award in 2012. His coauthored paper also received the Best Paper Award from the Asia-Pacific Signal and Information Processing Association Annual Summit and Conference (APSIPA ASC) in 2013. He is also a guest editor of EURASIP Special Issue on Cooperative MIMO Multicell Networks and of IJSNET Special Issue on Advances in Theory and Applications of Wireless, Ad Hoc, and Sensor Networks. 